\title{
CONSTRUCTING VARIATIONS OF HODGE STRUCTURE USING YANG-MILLS THEORY AND APPLICATIONS TO UNIFORMIZATION
}

\author{
CARLOS T. SIMPSON
}

\section{INTRODUCTION}

The fundamental group is one of the most basic topological invariants of a space. The aim of this paper is to present a method of constructing representations of fundamental groups in complex geometry, using techniques of partial differential equations. A representation of the fundamental group of a manifold is the same thing as a vector bundle over the manifold with a connection whose curvature vanishes, and this condition amounts to a differential equation. On the other hand, the natural objects of geometry over a complex manifold are the holomorphic vector bundles and holomorphic maps between them. We will adopt a philosophy based on algebraic geometry, that these holomorphic objects are understandable, and this leads us to try to produce flat connections starting from holomorphic data.

Briefly, the results are as follows. We solve the Yang-Mills equations on holomorphic vector bundles with interaction terms, over compact and some noncompact complex Kähler manifolds, yielding flat connections when certain Chern numbers vanish. An application in the compact case gives necessary and sufficient conditions for a variety to be uniformized by any particular bounded symmetric domain.

The first such construction was the theorem of Narasimhan and Seshadri relating holomorphic vector bundles and unitary connections on a curve. It was later extended to higher dimensions by Donaldson, Uhlenbeck, and Yau. Their work serves as a paradigm for what we will prove, so it is worth describing first. Let $X$ be a compact complex manifold. One can produce unitary connections using holomorphic vector bundles as follows. There is a natural operator $\bar{\partial}$ which reflects the holomorphic structure of a bundle $E$. Given a metric on $E$, there is an operator $\partial$ defined by the condition that the sum $D=\partial+\bar{\partial}$ is a connection which preserves the metric. The curvature of $D$ is a two-form $F=D^{2}$ with coefficients in the endomorphisms of $E$. The equation $F=0$ is usually overdetermined, but there is a natural intermediate equation, itself of

Received by the editors December 8, 1987.

1980 Mathematics Subject Classification (1985 Revision). Primary 58E15; Secondary 14F05, $32 \mathrm{M} 15,53 \mathrm{C} 05$. 
interest in mathematical physics. Assume that $X$ has a Kähler metric $\omega$. A metric on $E$ is called Hermitian-Yang-Mills if the component of its curvature in the direction of $\omega$ is a scalar multiple of the identity, an equation we write $\Lambda F=\lambda \cdot \mathrm{Id}$.

The condition needed to get a solution comes from a central concept in the theory of holomorphic bundles, the notion of stability defined by Mumford [24, 37]. A holomorphic bundle is stable if for every proper subsheaf $V \subset E$,

$$
\frac{\operatorname{deg}(V)}{\operatorname{rk}(V)}<\frac{\operatorname{deg}(E)}{\operatorname{rk}(E)}
$$

The degree of a bundle is the product of its first Chern class with the appropriate multiple of the cohomology class of $\omega$, so the notion of stability may depend on the choice of $\omega$. If this seems to take us from the realm of geometry, it should be pointed out that when $X$ is a projective variety the class of $\omega$ is the same as the hyperplane section, and degrees and Chern classes may then be calculated as intersections of algebraic cycles.

The fundamental theorem is that an irreducible holomorphic vector bundle has a Hermitian-Yang-Mills metric if and only if it is stable. This was proved for curves by Narasimhan-Seshadri [25], for surfaces by Donaldson [7], and in general by Uhlenbeck-Yau [30]. Donaldson gave a simplified proof for projective varieties in [8]. The application to constructing flat connections is based on the fact that if the Chern classes of $E$ vanish then any Hermitian-Yang-Mills metric is flat $[34,35,36]$. In fact only certain classes have to be checked: it is enough that $c_{1}(E)=0$ and $c_{2}(E) \cdot[\omega]^{n-2}=0$. The irreducible flat unitary connections are given by stable bundles with such Chern classes vanishing.

The origin of the present work was an attempt to parametrize certain other flat connections, the variations of Hodge structure defined by Griffiths. A complex variation of Hodge structure $[10,11,5]$ is a $C^{\infty}$ vector bundle $V$ with a decomposition $V=\bigoplus_{p+q=w} V^{p, q}$, a flat connection $D$ satisfying Griffiths' transversality condition

$$
D: V^{p, q} \rightarrow A^{0,1}\left(V^{p+1, q-1}\right) \oplus A^{1,0}\left(V^{p, q}\right) \oplus A^{0,1}\left(V^{p, q}\right) \oplus A^{1,0}\left(V^{p-1, q+1}\right),
$$

and a polarization. $A^{i, j}$ denotes differential forms of type $(i, j)$ on $X$, with coefficients in the indicated bundle. A polarization is a parallel Hermitian form which makes the Hodge decomposition orthogonal, and which on $V^{p, q}$ is positive definite if $p$ is even and negative definite if $p$ is odd. Griffiths came up with this definition to encapsulate some facts about the monodromy and variation of period matrices in holomorphic families of complex manifolds. When a variation of Hodge structure comes from a family of manifolds, there is a lattice in $V$ preserved by the connection, coming from the integral cohomology of the members of the family. Griffiths included the existence of such a lattice as part of his definition, but we leave that condition out. Dropping that requirement means that there are many more complex variations of Hodge structure, and in particular a variation can be perturbed or changed in a continuous family. This 
leads to the question of whether there is a naturally associated geometric object which can serve as a parameter.

After considering the set of infinitesimal deformations of a variation, it becomes evident that one should make the following definition. A system of Hodge bundles is a direct sum of holomorphic bundles $E^{p, q}$ together with maps $\theta: E^{p, q} \rightarrow E^{p-1, q+1} \otimes \Omega_{X}^{1}$ such that $\theta \wedge \theta=0$. From a variation of Hodge structure, a system of Hodge bundles is obtained in a natural way: different components of the connection $D$ give holomorphic structures $\bar{\partial}$ on the $V^{p, q}$, and holomorphic maps $\theta$. If a variation of Hodge structure comes from a family of varieties, the associated system of Hodge bundles is easier to calculate than the variation itself. Our aim is to see how to construct a variation of Hodge structure starting from a system of Hodge bundles. A variation gives a holomorphic map from the universal cover of $X$ to the classifying space for Hodge structures [10], which is sometimes a Hermitian symmetric space. Thus one application of the construction is to give a criterion for uniformization, which we will discuss below. This paper accomplishes the construction of variations of Hodge structure when $X$ is a compact Kähler manifold or a quasiprojective curve, and some progress is made in the higher dimensional noncompact case. For now, fix the assumption that $X$ is compact.

The basic idea is to develop the theory of Narasimhan-Seshadri, Donaldson, and Uhlenbeck-Yau to apply to this situation. Notice that the Hermitian form preserved by the flat connection of a variation is not in general positive definite, so the structure group is noncompact and the construction of unitary connections does not directly apply. However, a variation with $V=V^{0,0}$ is unitary, and a system of Hodge bundles with $E=E^{0,0}$ is just a holomorphic bundle, and with this in mind the previous results may be taken as a model. A metric on a system of Hodge bundles is a direct sum of metrics on the $E^{p, q}$, and any such metric gives rise to a connection which preserves the associated indefinite form. We try to solve the Hermitian-Yang-Mills equations $\Lambda F=\lambda$. Id. Again the geometric condition is that of stability, which is the same as before except that only subsystems of Hodge sheaves, in other words subobjects compatible with $\theta$ and the direct sum, are considered. The resulting theorem (Proposition 8.1) is that the irreducible complex variations of Hodge structure correspond exactly to the stable systems of Hodge bundles with $c_{1}(E)=0$ and $c_{2}(E) \cdot[\omega]^{n-2}=0$. When $X$ is a compact curve this theorem is due to Beilinson and Deligne. Generalizing [25] had yielded a partial result, that existence of variations is preserved in families of stable systems, at which point Deligne explained their stronger result to me; this conversation provided one of the starting points for trying to treat the higher dimensional case.

In a recent paper Hitchin treats a somewhat more general situation for bundles of rank 2 on a curve [14]. He looks at pairs consisting of a holomorphic bundle $E$ and a map $\theta: E \rightarrow E \otimes \Omega_{X}^{1}$ which he calls the Higgs field. He solves 
the Yang-Mills equations with this interaction term to establish a correspondence between stable pairs of degree zero and irreducible local systems of rank 2. We will adopt Hitchin's setting as the most general one for our treatment, and define a Higgs bundle on a complex manifold $X$ to be a pair $(E, \theta)$, usually also making the integrability requirement $\theta \wedge \theta=0$. In extending the previous work, the definition of stability of a Higgs bundle $E$ is made by considering sub-Higgs sheaves $F \subset E$, in other words subsheaves preserved by $\theta$. The analogue of the $\bar{\partial}$-operator on a holomorphic bundle is the operator containing all of the data of a Higgs bundle, namely $D^{\prime \prime}=\bar{\partial}+\theta$. For any choice of metric on $E$ there is an operator $D^{\prime}=\partial+\bar{\theta}$ with $\partial$ defined as before and $\bar{\theta}$ defined by $(\bar{\theta} e, f)=(e, \theta f)$. This yields a connection $D=D^{\prime}+D^{\prime \prime}$ which will not in general preserve the metric. The connection has a curvature $F$, and the Hermitian-Yang-Mills equation is once again $\Lambda F=\lambda \cdot \mathrm{Id}$. We may now state the main result of the paper. It occurs in $\S 3$ as Theorem 1 and Proposition 3.4.

Theorem. Every stable Higgs bundle $E$ has a Hermitian-Yang-Mills metric. If $\theta \wedge \theta=0$, and $c_{1}(E)=0$ and $c_{2}(E) \cdot[\omega]^{n-2}=0$ then the connection is flat.

The results on variations of Hodge structure follow from this, because a system of Hodge bundles is a Higgs bundle with the extra structure of a Hodge decomposition, and the connection constructed will be compatible with this extra structure.

In fact it turns out that all irreducible flat connections are constructed by this theorem, as shown in Hitchin's case in the postscript written by Donaldson [9]. In general Corlette [3] shows that any irreducible flat bundle admits a harmonic metric (and Donaldson's argument works too). The Siu-Bochner formula that Corlette uses in $\S 5$ of [3] implies that a flat bundle with harmonic metric comes from a Higgs bundle in the above way.

The technique used to prove the main theorem is a combination of the techniques of Donaldson [7, 8], and Uhlenbeck-Yau [30]. In $\S 5$ we define Donaldson's functional, which is an integral of secondary characteristic classes. We then give an estimate (5.3) for the size of a metric in terms of the functional, using the method of Uhlenbeck-Yau. This is where the stability assumption comes in. In $\S 6$ we construct a solution of the nonlinear heat equation, which in the limit approaches the desired metric because of the estimate from $\S 5$.

We come now to the question of uniformization. The simplest nontrivial example of a system of Hodge bundles of degree zero on a curve $X$ is obtained by choosing a square-root $\Omega_{X}^{1 / 2}$ of the canonical bundle, and setting

$$
E^{1,0}=\Omega_{X}^{1 / 2}, \quad E^{0,1}=\Omega_{X}^{-1 / 2}
$$

with the obvious isomorphism $\theta$. If the genus of $X$ is $g \geq 2$ then the degree of the only possible saturated subsystem, $E^{0,1}$, is $<0$ so $E$ is stable. Thus $E$ gives rise to a variation of Hodge structure. The classifying map for this variation is an isomorphism between the universal cover of $X$ and the unit disc, and 
the monodromy representation is one of the $2^{2 g}$ liftings of the representation in $P S U(1,1)$ given by the uniformization, to $\pi_{1}(X) \rightarrow S U(1,1)$.

One of the motivations for proving the main theorem in higher dimensions was the hope of similarly obtaining results about uniformization of higher dimensional varieties. We obtain a general result for compact Kähler manifolds by introducing the notions of principal variation of Hodge structure and system of Hodge bundles. These will be explained in $\S \S 8$ and 9 . Theorem 2 is that $X$ is uniformized by a Hermitian symmetric space if and only if a uniformizing principal system of Hodge bundles of the type corresponding to that space exists, is stable, and has a second Chern class equal to zero. If $X$ is projective then these conditions are algebraic, so a corollary is Kazhdan's theorem that the property of being uniformized is preserved by Galois conjugation.

The statement can be simplified in some cases. For questions of uniformization by the unit ball, one considers the system of Hodge bundles whose components are $\Omega_{X}^{1}$ and $\mathscr{O}_{X}$, with the obvious map $\theta$. If this system is stable then it satisfies the Bogomolov-Gieseker inequality

$$
\left(2 c_{2}(X)-\frac{n}{n+1} c_{1}(X)^{2}\right) \cdot[\omega]^{n-2} \geq 0
$$

and if equality holds, then $X$ is covered by the unit ball. When $X$ is a surface of general type, this yields a proof of the theorem of Yau and Miyaoka that $c_{1}^{2} \leq 3 c_{2}$ and if equality holds then the surface is uniformized by the ball. For products of discs one obtains the following statement: if the tangent bundle is a direct sum of line bundles of degrees $<0$, and if $\left(2 c_{2}(X)-c_{1}(X)^{2}\right) \cdot[\omega]^{n-2}=0$, then $X$ is covered by a product of copies of the unit disc.

Deligne-Beilinson and Hitchin gave proofs of the uniformization theorem for curves from their results. The uniformization results in $\S 9$ are similar to results already known by the technique of Kähler-Einstein metrics [31, 18].

So far we have been discussing the case of a compact complex manifold $X$. However it turns out that the proof of the theorem works as well for some noncompact $X$. The assumptions needed on $X$ and the growth of the metric $\omega$ are explained in $\S 2$. Essentially $X$ is a Zariski open subset of a compact manifold $\bar{X}$ and the metric $\omega$ extends smoothly to $\bar{X}$.

As before we start with a Higgs bundle $E$ on $X$, but now an additional set of initial data is needed, in the form of an initial metric on $E$ with $\Lambda F$ bounded. These initial data are necessary because knowledge of the analytic object $E$ alone does not uniquely determine the connection to be constructed. An initial metric determines growth conditions at infinity, and we consider other metrics which are bounded with respect to the initial one. It also allows us to define Chern classes of $E$ and of subsheaves of $E$, by integrating Chern forms obtained from the initial curvature. In particular there is a notion of stability. The main theorem is the same as before, that if $E$ is stable then there is a Hermitian-Yang-Mills metric in the class of metrics being considered, and if furthermore the determinant of $E$ is flat and $c_{2}(E) \cdot[\omega]^{n-2}=0$ then there 
is a flat metric bounded with respect to the initial one (Theorem 1, Proposition 3.4, and Corollary 3.6). The main points in extending the proof from the compact case are that Stokes' theorem still holds, allowing us to define Donaldson's functional; that the equivalence between $L^{1}$ and $C^{0}$ estimates can be obtained from the compactification $\bar{X}$; and that we can solve the heat equation with boundary conditions on exhausting compact subsets $X_{\varphi}$, then take the limit as $\varphi \rightarrow \infty$.

Unfortunately this theorem does not represent the final desired goal. Remember that we are seeking to relate flat or Hermitian-Yang-Mills connections to the natural holomorphic objects of complex geometry, preferably over a compact space. The choice of initial metric falls outside of this class of objects, so what remains to be done is to relate the initial metric to some extra holomorphic data on the completion $\bar{X}$. There are four related problems: to construct initial metrics; to characterize the metrics by data on an extension of the bundle to $\bar{X}$; to show that the metrics constructed represent all possible choices; and to calculate the Chern classes of $E$ and its subobjects in terms of the holomorphic data on the extension to $\bar{X}$, thereby analyzing the notion of stability. Once these are accomplished then the theory will be satisfactorily extended to noncompact varieties, and we can expect to get uniformization results as well. (The Kähler-Einstein technique for obtaining uniformization results has been partially extended to the noncompact case [17], but in this case also the choice of initial metric is not completely understood.)

The above program is carried out for the problem of constructing variations of Hodge structure on a noncompact curve, in $\S 10$. The results give an indication, albeit incomplete, of what the answer should look like in general. Suppose for example $X=\bar{X}-x$. Then a regular system of Hodge bundles on $X$ is defined to be a system of Hodge bundles $E$ on $X$ together with an extension of $E$ to $\bar{X}$ and a filtration of the fiber $E_{x}^{p, q}$ by subspaces $E_{\alpha}^{p, q}$ for $0 \leq \alpha<1$, such that $\theta: E_{\alpha} \rightarrow E_{\alpha} \otimes \Omega_{X}^{1}\langle\log x\rangle$. (The logarithmic differentials are those generated by $d z / z$ at $x$.) The space $E_{\alpha}$ represents sections whose norms grow like $|z|^{\alpha}$ in the initial metric near $x$. Theorem 3 states that these data on $\bar{X}$ characterize metrics on $E$ over $X$ with bounded curvature, and that the degree of $E$ or a subsystem is calculated by taking the indices $\alpha$ into account in an obvious way. The result is similar to but weaker than Schmid's norm estimates [27]. Our proof, which is simpler and more in keeping with the methods of this paper, uses the curvature properties of the classifying space for Hodge structures [12] used by Schmid, and work of Cornalba and Griffiths on growth properties of metrics [4]. Finally in Theorem 4 we combine this with Theorem 1 to obtain an equivalence between the regular systems of Hodge bundles which are direct sums of stable ones of degree zero, and the complex variations of Hodge structure on $X$.

Some of the results of this paper for compact $X$ have been announced in the note [29]. 
I would like to thank my advisor, W. Schmid, for his help and encouragement, and also the following people: P. Deligne, Y. T. Siu, S. T. Yau, J. Bernstein, D. Kazhdan, G. Laumon, K. Vilonen, T. Mrowka, H. Tsuji, K. Corlette, K. Uhlenbeck, and W. Goldman. I would like to thank the NSF and the Sloan foundation for graduate fellowship support.

\section{Assumptions on $X$}

Let $X$ be a complex manifold of dimension $n$, with a Kähler metric $\omega$. Let $\Lambda$ denote the adjoint of wedging with $\omega$. We will make the following assumptions:

Assumption 1. $X$ has finite volume.

Assumption 2. There exists an exhaustion function $\phi$ with $\Delta \phi$ bounded. Take $\phi \geq 0$.

In Proposition 3.5 and Corollary 3.6 we will need the stronger assumption that $\phi$ is plurisubharmonic and $\partial \bar{\partial} \phi$ is bounded, in other words

$$
0 \leq \sqrt{-1} \partial \bar{\partial} \phi \leq C \omega .
$$

Assumption 3. There is an increasing function $a:[0, \infty) \rightarrow[0, \infty)$ with $a(0)=$ 0 and $a(x)=x$ for $x>1$, such that if $f$ is a bounded positive function on $X$ with $\Delta(f) \leq B$ then

$$
\sup _{X}|f| \leq C(B) a\left(\int_{X}|f|\right) .
$$

Furthermore, if $\Delta(f) \leq 0$ then $\Delta(f)=0$.

These will be the only facts we will need, although the situations envisioned are those provided for by the following propositions.

Proposition 2.1. If $X$ is compact then it satisfies the above assumptions.

Proof. The first two assumptions are clear, so we just have to show the third, following Donaldson [8]. For future reference we will treat the case where $B$ is a function in $L^{p}$ for $p>n$ instead of just a constant. First work on a ball $U$ of radius 1 , and suppose $\Delta f \leq b$. Let $w$ be the function with $\Delta w=b$, and $\left.w\right|_{\partial U}=0$. The elliptic estimates together with $L_{2}^{p} \subset C^{0}$ give $w(0) \leq C\|b\|_{L^{p}}$. Subtract from $f$ the harmonic function with the same boundary values and apply the maximum principle to get

$$
f(0) \leq w(0)+\int_{\partial U} f \leq C\|b\|_{L^{p}}+\int_{\partial U} f .
$$

Dilating this to a ball $U(x, r)$ of radius $r$ on which $\Delta f \leq B$, we get

$$
f(x) \leq r^{2-2 n / p}\|B\|_{L^{p}}+\frac{1}{r^{2 n-1}} \int_{\partial U(x, r)} f .
$$


Given $R$ there will be some $r$ between $R$ and $2 R$ such that $R \int_{\partial U(x, r)} f \leq$ $\|f\|_{L^{1}(X)}$. Therefore since $f$ is positive,

$$
\sup |f| \leq C R^{2-2 n / p}\|B\|_{L^{p}}+\frac{1}{R^{2 n}}\|f\|_{L^{1}(X)} .
$$

If $\|f\|_{L^{1}} \leq R^{2 n+2-2 n / p}$ then $\sup |f| \leq C R^{2-2 n / p}\left(1+\|B\|_{L^{p}}\right)$, and in any case $\sup |f| \leq C\left(\|B\|_{L^{p}}+\|f\|_{L^{1}}\right)$.

Proposition 2.2. Suppose $X$ is a Zariski open subset of a smooth compact Kähler manifold $\bar{X}$, and suppose the metric $\omega$ is the restriction of a smooth metric on $\bar{X}$. Then the above assumptions hold for $(X, \omega)$. If $\bar{X}-X$ is an ample divisor then we may assume that $\phi$ is plurisubharmonic and $\partial \bar{\partial} \phi$ is bounded.

Proof. Assumption 1 is clear. Stratify $\bar{X}=X \cup \bigcup_{\alpha} Y_{\alpha}$ with $Y_{\alpha}$ smooth. Let $T_{\alpha}$ be the positive distribution given by $T_{\alpha}(g)=\int_{Y_{\alpha}} g$ and let $\phi_{\alpha}^{\alpha}$ be a solution to

$$
\Delta \phi_{\alpha}=T_{\alpha}-C_{\alpha}
$$

on $\bar{X}$. Here $C_{\alpha}=\operatorname{vol}\left(Y_{\alpha}\right) / \operatorname{vol}(X)$. We may assume $\phi_{\alpha}$ positive. Near each point of $Y_{\alpha}$, the function $\phi_{\alpha}$ is an exhaustion function for $X$. Therefore $\phi=\sum_{\alpha} \phi_{\alpha}$ is an exhaustion function for $X$, and its laplacian is a constant on $X$. If $Y=\bar{X}-X$ is an ample divisor, we can choose a smooth metric with positive curvature on the line bundle $L=\mathscr{O}(Y)$ over $\bar{X}$. Let $\phi=-\log |1|$. Then $\partial \bar{\partial} \phi$ is the curvature of $\left.L\right|_{X}$ so $0 \leq \sqrt{-1} \partial \bar{\partial} \phi \leq C \omega$.

To prove Assumption 3 it suffices to show that if $f$ is a bounded function on $X$ which satisfies $\Delta(f) \leq B$ then considered as a function on $\bar{X}$ it satisfies $\Delta(f) \leq B$ weakly (then use Proposition 2.1). In fact we will show this when $B$ is an $L^{1}$ function, not just a constant. Proceed by induction on the stratification, so we may assume that $Y=\bar{X}-X$ is smooth, of complex codimension $k$ say. We have to show that for a nonnegative function $\eta$ compactly supported on a small ball around a point on $Y$,

$$
\int f \Delta(\eta) \leq \int B \eta
$$

Let $\psi$ be the Greens function for the distribution $T_{Y}$, so $\psi(x)$ is approximately $d(x, Y)^{2-2 k}$ or $-\log d(x, Y)$ if $k=1$; we may assume $\psi \geq 0$; and $\Delta \psi=0$ on $X$. Note that this exists in the neighborhood where $\eta$ is supported. For each $N$ let $\psi_{N}$ be a truncation of $\psi$ so that $\psi_{N} \equiv N$ near $Y$. We may assume

$$
\int\left|\Delta \psi_{N}\right| \leq C
$$

and

Now

$$
\int\left|d \psi_{N}\right| \leq C
$$

$$
\int f\left(1-\frac{1}{N} \psi_{N}\right) \Delta \eta \rightarrow \int f \Delta \eta
$$


as $N \rightarrow \infty$ and on the other hand,

$$
\int f \Delta\left(\left(1-\frac{1}{N} \psi_{N}\right) \eta\right) \leq \int B\left(1-\frac{1}{N} \psi_{N}\right) \eta \rightarrow \int B \eta
$$

since $\left(1-\frac{1}{N} \psi_{N}\right) \eta$ is supported away from $Y$. The difference between these two is

$$
\int \frac{f \eta}{N} \Delta \psi_{N}-2 \int \frac{f}{N}\left(d \psi_{N}, d \eta\right) \rightarrow 0
$$

as $N \rightarrow \infty$, since $f$ is bounded. This proves the proposition.

Remark. The conclusion $\Delta f \leq B$ weakly holds under the assumption that $\frac{f}{\psi} \rightarrow 0$ as $x \rightarrow Y$ instead of the condition that $f$ is bounded. This is because we can assume $\Delta \psi_{N}$ and $d \psi_{N}$ are supported on $\psi(x) \leq 2 N$, so the supremum of $\frac{f}{N}$ on the support of $\Delta \psi_{N}$ or $d \psi_{N}$ goes to 0 as $N \rightarrow \infty$.

Proposition 2.3. If $Y$ is a finite unramified cover of $X$, with the pullback metric, and if the above assumptions hold for $Y$ then they hold for $X$.

Proof. Let $\phi_{X}=\operatorname{Tr}_{Y / X}\left(\phi_{Y}\right)$. The rest is clear.

Proposition 2.4. If $X$ is a quasiprojective curve and $\bar{X}$ is its completion, and if $\omega$ is a metric on $X$ which locally on $\bar{X}$ looks like $v(z) d z \wedge d \bar{z}$ with

$$
\int_{X}|v(z)|^{p}<\infty
$$

for some $p>1$ then the assumptions hold for $(X, \omega)$.

Proof. The volume is finite since $v$ is integrable. To construct the exhaustion function $\phi$, use a Greens function near each of the punctures, to get a function whose laplacian is compactly supported on $X$. To prove Assumption 3 , let $\Delta_{0}$ denote the laplacian with respect to the euclidean metric $d z \wedge d \bar{z}$ on $\bar{X}$ (near a puncture). Thus $\Delta_{0}=v \Delta$. If $f$ is a function with $\Delta(f) \leq B$ then $\Delta_{0}(f) \leq B v$ on $X$. The argument in Proposition 2.2 shows that $\Delta_{0}(f) \leq B v$ weakly on $\bar{X}$. Now proceed as in Proposition 2.1.

Remark. I do not think Assumption 3 is true if one assumes only that the metric is integrable; for example I do not think it is true for a metric which looks like the Poincaré metric at the punctures. However one could obtain a bound which is not uniform on $X$.

\section{Metrics on Higgs bundles}

Let $X$ be as above. Following [14] we define a Higgs bundle on $X$ to be a holomorphic vector bundle $E$ together with a map $\theta: E \rightarrow E \otimes \Omega_{X}^{1}$. Let $D^{\prime \prime}$ denote the operator $\bar{\partial}+\theta$. If $H$ is a metric on $E$ then we get an operator $\partial_{H}$ so that $d_{H}=\bar{\partial}+\partial_{H}$ is the metric connection on $E$, and we can define $\bar{\theta}_{H}$ by

$$
(\theta u, v)_{H}=\left(u, \bar{\theta}_{H} v\right)_{H} .
$$


Set

and then

$$
D_{H}^{\prime}=\partial_{H}+\bar{\theta}_{H}
$$

$$
D_{H}=D^{\prime \prime}+D_{H}^{\prime}
$$

is a connection on $E$. Let $F_{H}$ denote its curvature, and let $F_{H}^{\perp}$ denote the trace-free part of $F_{H}$.

Lemma 3.1. The following formulas from the usual case still hold:

(a) $\sqrt{-1}\left[\Lambda, D^{\prime \prime}\right]=\left(D_{H}^{\prime}\right)^{*}$ and $\sqrt{-1}\left[\Lambda, D_{H}^{\prime}\right]=-\left(D^{\prime \prime}\right)^{*}$;

(b) if $s$ and $h$ are selfadjoint sections of $\operatorname{End}(E)$ then

$$
\left|D^{\prime \prime}(s) h\right|_{H}^{2}=-\sqrt{-1} \Lambda \operatorname{Tr}\left(D^{\prime \prime}(s) \cdot h^{2} D_{H}^{\prime} s\right) ;
$$

(c) if $H=K h$ for a positive selfadjoint $h$ then

$$
\begin{gathered}
D_{H}^{\prime}=D_{K}^{\prime}+h^{-1} D_{K}^{\prime}(h) \\
\Delta_{K}^{\prime}(h)=h \sqrt{-1}\left(\Lambda F_{H}-\Lambda F_{K}\right)+\sqrt{-1} \Lambda D^{\prime \prime}(h) h^{-1} D_{K}^{\prime}(h)
\end{gathered}
$$

where the laplacian is $\Delta^{\prime}=\left(D^{\prime}\right)^{*} D^{\prime}=\sqrt{-1} \Lambda D^{\prime \prime} D^{\prime}$; and

(d) if $H=K h$ then

$$
\Delta \log \operatorname{Tr}(h) \leq 2\left(\left|\Lambda F_{H}\right|_{H}+\left|\Lambda F_{K}\right|_{K}\right) .
$$

In (c) and (d), if $\operatorname{det}(h)=1$ then the curvatures can be replaced by the trace-free curvatures $F^{\perp}$.

Proof. Write $\theta=\sum A_{i} d z_{i}$ in normal coordinates. Then $\bar{\theta}_{H}=\sum A_{i}^{*} d \bar{z}_{i}$ and $\sqrt{-1}\left[\Lambda, d z_{i}\right]=\left(d \bar{z}_{i}\right)^{*}$ which gives (a). For (b) note that if $s$ is selfadjoint then $\left[A_{i}^{*}, s\right]=-\left[A_{i}, s\right]^{*}$ so

$$
\begin{aligned}
-\sqrt{-1} \Lambda \operatorname{Tr}\left(\left[A_{i}, s\right] d z_{i} h^{2} \wedge\left[A_{i}^{*}, s\right] d \bar{z}_{i}\right) & =2 \operatorname{Tr}\left(\left[A_{i}, s\right] h\left(\left[A_{i}, s\right] h\right)^{*}\right) \\
& =\left|A_{i}(s) h d z_{i}\right|^{2} .
\end{aligned}
$$

For the first part of (c),

$$
\bar{\theta}_{H}=h^{-1} \bar{\theta}_{K} h=\bar{\theta}_{K}+h^{-1}\left[\bar{\theta}_{K}, h\right] .
$$

The second formula follows from the first, using $\Lambda F=\Lambda\left(D^{\prime \prime} D^{\prime}+D^{\prime} D^{\prime \prime}\right)$.

Now we show (d). Take the trace of the formula of part (c), and use the facts that $D$ commutes with $\operatorname{Tr}$, and $h$ is pointwise positive definite, to get

$$
\Delta_{\partial} \operatorname{Tr}(h) \leq \operatorname{Tr}(h)\left(\left|\Lambda F_{H}\right|_{H}+\left|\Lambda F_{K}\right|_{K}\right)+\sqrt{-1} \Lambda \operatorname{Tr} D^{\prime \prime}(h) h^{-1} D_{K}^{\prime}(h) .
$$

The last term is equal to $-\left|D^{\prime \prime}(h) h^{-1 / 2}\right|_{K}^{2}$ so

$$
\Delta_{\partial} \operatorname{Tr}(h)+\left|D^{\prime \prime}(h) h^{-1 / 2}\right|_{K}^{2} \leq \operatorname{Tr}(h)\left(\left|\Lambda F_{H}\right|_{H}+\left|\Lambda F_{K}\right|_{K}\right) .
$$

On the other hand,

$$
\operatorname{Tr}(h) \Delta_{\bar{\partial}} \log \operatorname{Tr}(h)=\Delta_{\bar{\partial}} \operatorname{Tr}(h)+\frac{|\bar{\partial} \operatorname{Tr}(h)|^{2}}{\operatorname{Tr}(h)}
$$


The last term in this equation is

$$
\frac{\left|\operatorname{Tr}\left(D^{\prime \prime}(h) h^{-1 / 2} h^{1 / 2}\right)\right|^{2}}{\left|h^{1 / 2}\right|_{K}^{2}} \leq\left|D^{\prime \prime}(h) h^{-1 / 2}\right|_{K}^{2}
$$

To see this note that in general $\left|\sum_{i} A_{i} b_{i}\right|^{2} \leq \sum_{i}\left|A_{i}\right|^{2} \sum_{i}\left|b_{i}\right|^{2}$ for vectors $A_{i}$ and scalars $b_{i}$; then choose an orthonormal basis of eigenvectors for $h$, so $h^{-1 / 2}=$ $\operatorname{diag}\left(b_{i}\right)$, and let $A_{i}$ be the diagonal elements (one-forms) of $D^{\prime \prime}(h) h^{-1 / 2}$. Therefore

$$
\operatorname{Tr}(h) \Delta_{\bar{\partial}} \log \operatorname{Tr}(h) \leq \Delta_{\bar{\partial}} \operatorname{Tr}(h)+\left|D^{\prime \prime}(h) h^{-1 / 2}\right|_{K}^{2} .
$$

The proof is concluded using the fact that $\Delta=2 \Delta_{\bar{\partial}}=2 \Delta_{\partial}$ on scalar functions. Donaldson, in [8], used an identical estimate for the laplacian of the highest eigenvalue of $\log (h)$. The above version was suggested by Y. T. Siu.

Now suppose $(E, \theta)$ is a Higgs bundle on $X$, with a background metric $K$. Make the assumption

$$
\sup _{X}\left|\Lambda F_{K}\right| \leq B
$$

Define the degree of $E$ to be the real number

$$
\operatorname{deg}(E, K)=\sqrt{-1} \int_{X} \operatorname{Tr}\left(\Lambda F_{K}\right)
$$

A sub-Higgs sheaf of $E$ is an analytic subsheaf $V \subset E$ such that $\theta: V \rightarrow$ $V \otimes \Omega_{X}^{1}$. If $V$ is a saturated subsheaf then outside of codimension 2 it is a subvector bundle. Let $\pi$ denote the projection onto $V$ using the metric $K$; it is defined almost everywhere. The metric $K$ restricts to a metric on $V$ outside codimension 2 , so we can define the degree by integrating outside codimension 2.

Lemma 3.2 (Chern-Weil formula).

$$
\operatorname{deg}(V, K)=\sqrt{-1} \int_{X} \operatorname{Tr}\left(\pi \Lambda F_{K}\right)-\int_{X}\left|D^{\prime \prime} \pi\right|_{K}^{2} .
$$

This is either a real number or $-\infty$. If the degree is not $-\infty$ then $\pi \in L_{1}^{2}$. If $X$ is compact then this is the degree of the sheaf $V$.

Proof. $D_{V}^{\prime}=\pi D^{\prime} \pi$ and $D_{V}^{\prime \prime}=\pi D^{\prime \prime} \pi$. These imply

$$
\Lambda F_{V}=\pi \Lambda F \pi+\Lambda D^{\prime \prime}(\pi) D^{\prime}(\pi)
$$

which gives the desired formula. One checks that the first Chern form defined by the metric where $V$ is a bundle is a closed current on $X$. It represents the first Chern class of $V$ outside of codimension 2, and hence on all of $X$. Thus if $X$ is compact the above integral is the product of the first Chern class of $V$ with $[\omega]^{n-1}$ as claimed. 
Let $A$ be a group acting by holomorphic automorphisms of $X$ preserving the metric $\omega$ and acting compatibly by automorphisms $\alpha: E \rightarrow E$ which preserve the metric $K$ and act on $\theta$ by homotheties:

$$
\alpha \theta \alpha^{-1}=\lambda(\alpha) \theta .
$$

Note that $|\lambda(\alpha)|=1$ and

$$
\alpha \bar{\theta}_{K} \alpha^{-1}=\overline{\lambda(\alpha)} \bar{\theta}_{K}
$$

so $\Lambda F_{K}=\Lambda\left(d_{K}^{2}+\theta \bar{\theta}_{K}+\bar{\theta}_{K} \theta\right)$ is preserved by $A$.

We say that $(E, K)$ is stable if for every proper saturated sub-Higgs sheaf $V \subset E$ which is preserved by $A$,

$$
\frac{\operatorname{deg}(V, K)}{\operatorname{rk}(V)}<\frac{\operatorname{deg}(E, K)}{\operatorname{rk}(E)} .
$$

We can now state our main theorem. The proof will be given in the next four sections.

Theorem 1. Let $(X, \omega)$ satisfy the assumptions of $\S 2$, and suppose $E$ is a Higgs bundle with metric $K$ satisfying the assumption that $\sup \left|\Lambda F_{K}\right|<\infty$. Suppose $(E, K)$ is stable with respect to a group $A$ acting as above. Then there is a metric $H$ with $\operatorname{det}(H)=\operatorname{det}(K), H$ and $K$ mutually bounded, $D^{\prime \prime}\left(K^{-1} H\right) \in L^{2}$, and $H$ invariant under $A$, such that

$$
\Lambda F_{H}^{\perp}=0 .
$$

A metric whose curvature satisfies the condition $\Lambda F_{H}^{\perp}=0$ is called Hermitian-Yang-Mills. There is a converse to Theorem 1.

Proposition 3.3. If $E$ is a Higgs bundle with metric $H$ such that

$$
\Lambda F_{H}^{\perp}=0
$$

then for any proper saturated sub-Higgs sheaf $V \subset E$,

$$
\frac{\operatorname{deg}(V, H)}{\mathrm{rk}(V)} \leq \frac{\operatorname{deg}(E, H)}{\mathrm{rk}(E)}
$$

and if equality holds then $E=V \oplus V^{\perp}$ is an orthogonal direct sum of sub-Higgs bundles.

Proof. It is obvious from the Chern-Weil formula $[19,34]$.

In the remainder of the paper we will give applications. Most of these will be constructions of flat connections.

Proposition 3.4 (Bogomolov-Gieseker inequality). Suppose E is a Higgs bundle on $X$ such that

$$
\theta^{2}=0: E \rightarrow E \otimes \Omega_{X}^{2} .
$$

Suppose $H$ is a metric with $\Lambda F_{H}^{\perp}=0$. Then

$$
\left(2 c_{2}(E, H)-\frac{r-1}{r} c_{1}(E, H)^{2}\right)[\omega]^{n-2}=C \int_{X} \operatorname{Tr}\left(F_{H}^{\perp} \wedge F_{H}^{\perp}\right) \wedge \omega^{n-2} \geq 0
$$


and if equality holds then $F_{H}^{\perp}=0$. In particular if $\operatorname{Tr}\left(F_{H}\right)=0$ and $c_{2}(E, H)[\omega]^{n-2}=0$ then the connection $D$ is flat.

Proof. See [30, 34, 35]. If $\theta^{2}=0$ then the curvature is

$$
F=d^{2}+\bar{\partial}(\bar{\theta})+\partial(\theta)+\theta \bar{\theta}+\bar{\theta} \theta .
$$

The component $F^{1,1}=d^{2}+\theta \bar{\theta}+\bar{\theta} \theta$ consists of forms of type $(1,1)$ on the base, and $F^{1,1}=\overline{F^{1,1}}$ refering to complex conjugation in End $E$ with respect to the metric. The Riemann bilinear relations [32] imply that

$$
\operatorname{Tr}\left(F^{1,1} \wedge F^{1,1}\right) \omega^{n-2}=C\left(\left|F^{1,1}-\frac{1}{n} \omega \Lambda F\right|^{2}-|\Lambda F|^{2}\right) \omega^{n} .
$$

On the other hand, $\bar{\theta}$ is the negative of the complex conjugate of $\theta$ with respect to the real structure on End $E$ induced by the metric so the Riemann bilinear relations for forms of type $(2,0)$ imply

$$
\operatorname{Tr}(\bar{\partial}(\bar{\theta}) \wedge \partial(\theta)) \omega^{n-2}=C|\partial(\theta)|^{2} \omega^{n} .
$$

The constant is the same so

$$
\operatorname{Tr}(F \wedge F) \omega^{n-2}=C\left(\left|F-\frac{1}{n} \omega \Lambda F\right|^{2}-|\Lambda F|^{2}\right) \omega^{n} .
$$

Similarly for $F^{\perp}$.

If $X$ is not compact, then the Chern classes measured with different metrics need not be equal a priori. We get the following statement suggested by $\mathrm{S}$. T. Yau.

Proposition 3.5. Suppose our exhaustion function $\phi$ satisfies $0 \leq \sqrt{-1} \partial \bar{\partial} \phi \leq$ $C \omega$. Suppose $E$ is a Higgs bundle with $\theta^{2}=0$, with an initial metric $K$ such that $\sup \left|\Lambda F_{K}\right|<\infty$. Suppose $E$ is stable. Then for the metric $H$ constructed in Theorem 1,

$$
\int_{X} \operatorname{Tr}\left(F_{H} \wedge F_{H}\right) \omega^{n-2} \leq \int_{X} \operatorname{Tr}\left(F_{K} \wedge F_{K}\right) \omega^{n-2} .
$$

The same holds for $F^{\perp}$ since $\operatorname{det}(H)=\operatorname{det}(K)$.

The proof will be given at the end of $\S 7$. Combining Theorem 1 with Propositions 3.4 and 3.5 we get

Corollary 3.6. Suppose $0 \leq \sqrt{-1} \partial \bar{\partial} \phi \leq C \omega$ and let $E$ be a Higgs bundle with $\theta^{2}=0$, with a metric such that $\sup \left|\Lambda F_{K}\right|<\infty, \operatorname{det}(K)$ flat, $c_{2}(E, K)[\omega]^{n-2}=$ 0 and $E$ stable with respect to $K$ and the action of some group $A$ as before. Then there is a flat $A$-invariant metric $H$ comparable to $K$.

\section{SOME CONSTRUCTIONS INVOLVING HERMITIAN MATRICES}

In this section we will review some constructions involving hermitian matrices. Let $E$ be a Higgs bundle with a fixed metric $K$, and let $S=S(E)$ denote 
the real vector bundle of selfadjoint endomorphisms of $E$. Suppose $\varphi: \mathbf{R} \rightarrow \mathbf{R}$ is a smooth function. Then we define a smooth map of fiber bundles over $X$

$$
\varphi: S \rightarrow S
$$

as follows: suppose $s \in S$, then at each point in $X$, choose an orthonormal basis $\left\{e_{i}\right\}$ for $E$ with $s\left(e_{i}\right)=\lambda_{i} e_{i}$, and set

$$
\varphi(s)\left(e_{i}\right)=\varphi\left(\lambda_{i}\right) e_{i} .
$$

Suppose $\Psi: \mathbf{R} \times \mathbf{R} \rightarrow \mathbf{R}$ is a smooth function of two variables. Then we define a map of fiber bundles

$$
\Psi: S \rightarrow S(\text { End } E),
$$

where $S($ End $E)$ consists of elements of $\operatorname{End}(\operatorname{End} E)$ which are selfadjoint with respect to the metric $\operatorname{Tr}\left(A B^{*}\right) . \Psi$ is described as follows. Suppose $s \in S$ and $A \in \operatorname{End}(E)$. Choose an orthonormal basis $\left\{e_{i}\right\}$ of eigenvectors of $s$ with eigenvalues $\lambda_{i}$. Let $\left\{\hat{e}_{i}\right\}$ be the dual basis in $E^{*}$, and write $A=\sum_{i, j} A_{i j} \hat{e}_{i} \otimes e_{j}$. Then set

$$
\Psi(s)(A)=\sum_{i, j} \Psi\left(\lambda_{i}, \lambda_{j}\right) A_{i j} \hat{e}_{i} \otimes e_{j} .
$$

Again this is well defined, smooth in $s$ and over $X$, and linear in $A$.

If the functions $\varphi$ and $\Psi$ are analytic, then we can express the constructions above as power series. If

$$
\varphi(\lambda)=\sum a_{n} \lambda^{n}
$$

then

$$
\varphi(s)=\sum a_{n} s^{n}
$$

If

$$
\Psi\left(\lambda_{1}, \lambda_{2}\right)=\sum b_{m n} \lambda_{1}^{m} \lambda_{2}^{n}
$$

then

$$
\Psi(s)(A)=\sum b_{m n} s^{n} A s^{m} .
$$

The construction $\Psi$ gives a convenient way of expressing the derivatives of the construction $\varphi$. More precisely, suppose $\varphi: \mathbf{R} \rightarrow \mathbf{R}$ is a smooth function. Define $d \varphi: \mathbf{R} \times \mathbf{R} \rightarrow \mathbf{R}$ by

$$
d \varphi\left(\lambda_{1}, \lambda_{2}\right)=\frac{\varphi\left(\lambda_{1}\right)-\varphi\left(\lambda_{2}\right)}{\lambda_{1}-\lambda_{2}},
$$

which is taken as $(d \varphi / d \lambda)\left(\lambda_{1}\right)$ if $\lambda_{1}=\lambda_{2}$. If $s \in S$, then

$$
D^{\prime \prime} \varphi(s)=d \varphi(s)\left(D^{\prime \prime} s\right)
$$

where the right side uses the obvious extension to form-coefficients in the second variable. To see this for example when $\varphi$ is analytic, note that if $\varphi(\lambda)=\lambda^{n}$ then

$$
d \varphi\left(\lambda_{1}, \lambda_{2}\right)=\sum_{i+j=n-1} \lambda_{1}^{i} \lambda_{2}^{j}
$$


whereas

$$
D^{\prime \prime}\left(s^{n}\right)=\sum_{i+j=n-1} s^{i} D^{\prime \prime}(s) s^{j} .
$$

The constructions $\varphi(s)$ and $\Psi(s)$ retain the same positivity properties as $\varphi$ and $\Psi$. For example if $\varphi(\lambda)>0$ for all $\lambda$, then $\varphi(s)$ is positive definite for all $s$. And if $\Psi\left(\lambda_{1}, \lambda_{2}\right)>0$ for all $\lambda_{1}, \lambda_{2}$ then $\operatorname{Tr}\left(\Psi(s)(A) A^{*}\right)>0$ for all $s$ and all $A \in \operatorname{End}(E)$.

We will describe how these constructions behave on Sobolev spaces. Fix a smooth metric $K$ on a Higgs bundle $E$. Using the metric we can define the space $L^{p}(S)$ of sections of the real bundle $S$. Let $L_{1}^{p}(S)$ denote the space of sections $s$ such that $s \in L^{p}$ and $D^{\prime \prime} s \in L^{p}$. Note that this is a condition on the derivative $\bar{\partial} s$ and also a growth condition involving $\theta$ if $X$ is noncompact. For a given number $b$ denote the closed subspaces of sections $s$ with $|s| \leq b$ by $L_{0, b}^{p}$ and $L_{1, b}^{p}$. Finally let $P(S)$ denote the normed space of smooth sections $s \in S$ with norm

$$
\|s\|_{P}=\sup _{X}|s|+\left\|D^{\prime \prime} s\right\|_{L^{2}}+\left\|\Delta_{K^{\prime}}^{\prime} s\right\|_{L^{1}}
$$

The constructions $\varphi$ and $\Psi$ behave in a slightly delicate fashion on $L^{p}$ and $L_{1}^{p}$ as is shown in the following proposition (c.f. [26]). They behave better on $P$ since the $C^{0}$ norm is controlled.

Proposition 4.1. Let $\varphi$ and $\Psi$ be functions as above.

(a) The map $\varphi$ extends to a continuous nonlinear map

$$
\varphi: L_{0, b}^{p}(S) \rightarrow L_{0, b^{\prime}}^{p}(S)
$$

for some $b^{\prime}$.

(b) The map $\Psi$ extends to a map

$$
\Psi: L_{0, b}^{p}(S) \rightarrow \operatorname{Hom}\left(L^{p}(\text { End } E), L^{q}(\text { End } E)\right)
$$

for $q \leq p$, and for $q<p$ it is continuous in the operator norm topology.

(c) The map $\varphi$ extends to a map

$$
\varphi: L_{1, b}^{p}(S) \rightarrow L_{1, b^{\prime}}^{q}(S)
$$

for $q \leq p$, and it is continuous for $q<p$. The formula $D^{\prime \prime} \varphi(s)=$ $d \varphi(s)\left(D^{\prime \prime} s\right)$ holds in this context.

(d) If $\varphi$ and $\Psi$ are analytic with infinite radius of convergence, then the maps

$$
\begin{gathered}
\varphi: P(S) \rightarrow P(S), \\
\Psi: P(S) \rightarrow P(\operatorname{End}(\operatorname{End}(E)))
\end{gathered}
$$

are analytic.

Proof. If $\varphi$ and $\Psi$ are functions as in the proposition, and if a bound $\left|s_{i}\right| \leq b$ is fixed, then there are constants so that

$$
\left|\varphi\left(s_{1}\right)-\varphi\left(s_{2}\right)\right| \leq C\left|s_{1}-s_{2}\right|
$$


and

$$
\left|\Psi\left(s_{1}\right)(A)-\Psi\left(s_{2}\right)(A)\right| \leq C\left|s_{1}-s_{2}\right| \cdot|A|
$$

pointwise on $X$. From this, (a) is clear. To prove (b) note that $\left\|s_{1}-s_{2}\right\|_{L^{r}} \leq$ $C\left\|s_{1}-s_{2}\right\|_{L^{p}}$ since $\left|s_{1}-s_{2}\right| \leq 2 b$. For any $q<p$ we can choose $r$ so that $L^{r} \cdot L^{p} \subset L^{q}$ so

$$
\left\|\Psi\left(s_{1}\right)(A)-\Psi\left(s_{2}\right)(A)\right\|_{L^{q}} \leq C\left\|s_{1}-s_{2}\right\|_{L^{p}}\|A\|_{L^{p}} .
$$

To prove (c), apply part (b) to $d \varphi$. Note that

$$
D^{\prime \prime} \varphi\left(s_{1}\right)-D^{\prime \prime} \varphi\left(s_{2}\right)=d \varphi\left(s_{1}\right)\left(D^{\prime \prime}\left(s_{1}-s_{2}\right)\right)+\left(d \varphi\left(s_{1}\right)-d \varphi\left(s_{2}\right)\right)\left(D^{\prime \prime} s_{2}\right)
$$

so

$$
\left\|D^{\prime \prime} \varphi\left(s_{1}\right)-D^{\prime \prime} \varphi\left(s_{2}\right)\right\|_{L^{q}} \leq b^{\prime}\left\|s_{1}-s_{2}\right\|_{L_{1}^{p}}+C\left\|s_{1}-s_{2}\right\|_{L^{p}}\left\|s_{2}\right\|_{L_{1}^{p}} .
$$

(d) Suppose $\varphi$ and $\Psi$ are analytic. Then the constructions are expressed in power series, so we just have to show that

$$
\left\|s^{m}\right\|_{P} \leq C^{m}\|s\|_{P}^{m} \text {. }
$$

But pointwise on $X$,

$$
\left|D^{\prime \prime} s^{m}\right| \leq m|s|^{m-1}\left|D^{\prime \prime} s\right|,
$$

$$
\left|\Lambda D^{\prime \prime} D_{K}^{\prime} s^{m}\right| \leq m|s|^{m-1}\left|\Lambda D^{\prime \prime} D_{K}^{\prime} s\right|+m(m-1)|s|^{m-2}\left|D^{\prime \prime} s\right|\left|D_{K}^{\prime} s\right|
$$

and $\left|D_{K}^{\prime} s\right|=\left|D^{\prime \prime} s\right|$ since $s$ is selfadjoint. These imply the desired inequality.

\section{DONALDSON'S FUNCTIONAL}

Let $E$ be a Higgs bundle over $X$. Let $\mathscr{P}$ denote the space of smooth metrics $K$ such that

$$
\int_{X}\left|\Lambda F_{K}\right|_{K}<\infty
$$

Due to the last part of Proposition 4.1, we can make $\mathscr{P}$ into an analytic manifold with local charts

$$
\begin{gathered}
P\left(S_{H}\right) \rightarrow \mathscr{P}, \\
s \mapsto H e^{s} .
\end{gathered}
$$

Divide $\mathscr{P}$ into components such that each of these charts covers a component. Recall that $P\left(S_{H}\right)$ is the space of smooth sections $s$ with norm

$$
\|s\|_{P}=\sup _{X}|s|+\left\|D^{\prime \prime} s\right\|_{L^{2}}+\left\|\Delta^{\prime} s\right\|_{L^{1}} .
$$

Let $\mathscr{P}_{0}$ be one of the components. For two metrics in this component $K$ and $H=K e^{s}$, define

$$
M(K, H)=\sqrt{-1} \int_{X} \operatorname{Tr}\left(s \Lambda F_{K}\right)+\int_{X}\left(\Psi(s)\left(D^{\prime \prime} s\right), D^{\prime \prime} s\right)_{K}
$$

where $\Psi$ is constructed as in the previous section from the function

$$
\Psi\left(\lambda_{1}, \lambda_{2}\right)=\frac{e^{\lambda_{2}-\lambda_{1}}-\left(\lambda_{2}-\lambda_{1}\right)-1}{\left(\lambda_{2}-\lambda_{1}\right)^{2}} .
$$


This is the functional used by Donaldson in [7,8]. $M(K, H)$ is analytic in $K$ and $H$, since $K_{1} \mapsto \Lambda F_{K_{1}} \in L^{1}$ is analytic with respect to the norm using a fixed $K_{0}$ by Lemma 3.1.

Proposition 5.1. If $K, H$, and $J$ are three metrics in the same component $\mathscr{P}_{0}$, then

$$
M(K, H)+M(H, J)=M(K, J) .
$$

Proof. Donaldson defined $M$ by a variational formula [7], and then in [8] derived the above formula. Both steps require integration by parts, so we have to be careful if $X$ is not compact. To prove the proposition it suffices to show that

$$
\frac{\partial^{2}}{\partial t_{1} \partial t_{2}} M\left(K e^{t_{1} s_{1}}, K e^{t_{1} s_{1}} e^{t_{2} s_{2}}\right)=\frac{\partial^{2}}{\partial t_{1} \partial t_{2}} M\left(K, K e^{t_{1} s_{1}} e^{t_{2} s_{2}}\right)
$$

at $t_{1}=t_{2}=0$, and

$$
\frac{\partial^{2}}{\partial t^{2}} M\left(K, K e^{t s}\right)=\frac{\partial^{2}}{\partial t \partial u} M\left(K e^{t s}, K e^{(t+u) s}\right)
$$

at $u=0, t=1$. In the first, the left side is

$$
\frac{\partial}{\partial t_{1}} \int_{X} \sqrt{-1} \operatorname{Tr}\left(s_{2} \Lambda F_{K e^{t_{1} s_{1}}}\right)=\sqrt{-1} \int_{X} \operatorname{Tr}\left(s_{2} \Lambda D^{\prime \prime} D_{K}^{\prime}\left(s_{1}\right)\right)
$$

while the right side is

$$
\int_{X}\left(D^{\prime \prime} s_{1}, D^{\prime \prime} s_{2}\right)=-\sqrt{-1} \int_{X} \Lambda \operatorname{Tr}\left(D^{\prime \prime} s_{2} \wedge D_{K}^{\prime} s_{1}\right)
$$

(note that $\Psi(0)=\frac{1}{2}$ ). These differ by

$$
\sqrt{-1} \int_{X} \bar{\partial} \operatorname{Tr}\left(s_{2} \partial_{K} s_{1}\right) \wedge \omega^{n-1} .
$$

For the second identity, the left side is

$$
\int_{X}\left(\Psi_{1}(s)\left(D^{\prime \prime} s\right), D^{\prime \prime} s\right)_{K}
$$

where

$$
\Psi_{1}\left(\lambda_{1}, \lambda_{2}\right)=\frac{\partial^{2}}{\partial t^{2}}\left(t^{2} \Psi\left(t \lambda_{1}, t \lambda_{2}\right)\right)=e^{\lambda_{2}-\lambda_{1}} .
$$

Thus the left side becomes

$$
\int_{X}\left(D^{\prime \prime} s, D^{\prime \prime} s\right)_{K e^{s}}=-\sqrt{-1} \int_{X} \Lambda \operatorname{Tr}\left(D^{\prime \prime} s \wedge D_{K e^{s}}^{\prime} s\right) .
$$

The right side is

$$
\sqrt{-1} \int_{X} \Lambda \operatorname{Tr}\left(s D^{\prime \prime} D_{K e^{s}}^{\prime} s\right)
$$

so they differ by

$$
\sqrt{-1} \int_{X} \bar{\partial} \operatorname{Tr}\left(s \partial_{K e^{s}} s\right) \wedge \omega^{n-1}
$$


Thus the following lemma (Stokes' theorem for $X$ ) will complete the proof of the proposition. It is clear if $X$ is compact.

Lemma 5.2. Suppose $X$ has an exhaustion function $\phi$ with $\int_{X}|\Delta \phi|<\infty$, and suppose $\eta$ is a $(2 n-1)$-form with $\int_{X}|\eta|^{2}<\infty$. Then if $d \eta$ is integrable,

$$
\int_{X} d \eta=0
$$

Proof. Take $\phi \geq 0$. Let $X_{t}$ be the set where $\phi(x) \leq t$ and let $Y_{t}$ be the boundary $\phi(y)=t$. By Stokes' theorem

$$
\int_{X_{t}}|d \phi|^{2}=\int_{X_{t}}(t-\phi) \Delta \phi \leq C t .
$$

On the other hand,

so

$$
\frac{d}{d t} \int_{X_{t}}|d \phi|^{2}=\int_{Y_{t}}|d \phi|
$$

$$
\int_{0}^{T} \int_{Y_{t}}|d \phi| d t \leq C T
$$

Stokes' theorem for the form $\eta$ is

$$
\int_{X_{t}} d \eta=\int_{Y_{t}} \eta
$$

and the $L^{2}$ condition is

$$
\int_{0}^{T} \int_{Y_{t}} \frac{|\eta|^{2}}{|d \phi|} d t \leq C
$$

Now

$$
\begin{aligned}
\int_{0}^{T}\left|\int_{X_{t}} d \eta\right| d t & \leq \int_{0}^{T} \int_{Y_{t}}|\eta| d t \\
& \leq\left(\int_{0}^{T} \int_{Y_{t}} \frac{|\eta|^{2}}{|d \phi|}\right)^{1 / 2}\left(\int_{0}^{T} \int_{Y_{t}}|d \phi|\right)^{1 / 2} \\
& \leq C T^{1 / 2} .
\end{aligned}
$$

Therefore there is a subsequence $t_{i} \rightarrow \infty$ such that

$$
\int_{X_{t_{i}}} d \eta \rightarrow 0
$$

Now we can give the main estimate involving $M$.

Proposition 5.3. Fix a number $B$. Let $E$ be a Higgs bundle over $X$, with a metric $K$ such that $\sup _{X}\left|\Lambda F_{K}\right| \leq B$. Suppose a group $A$ acts as before. Suppose $E$ is stable with respect to the metric $K$ and the action of $A$. Then there are constants such that

$$
\sup _{X}|s| \leq C_{1}+C_{2} M\left(K, K e^{s}\right)
$$


for any $A$-invariant selfadjoint endomorphism $s \in P(S)$ with $\operatorname{Tr}(s)=0$, such that $\sup _{X}|s|<\infty$ and such that $\sup \left|\Lambda F_{K e^{s}}\right| \leq B$.

Proof. We will use the method of Uhlenbeck-Yau [30] to show that if the estimate does not hold, there is a sub-Higgs sheaf contradicting stability. First of all, by Lemma 3.1(d), Assumption 3, and the hypothesis that $\sup \left|\Lambda F_{K}\right|$, $\sup _{X}\left|\Lambda F_{K e^{s}}\right| \leq B$,

$$
\sup _{X}|s| \leq C_{1}+C_{2}\|s\|_{L^{1}}
$$

for the sections $s$ we consider. Suppose the required estimate does not hold. We can find $s$ contradicting the estimate with $\|s\|_{L^{1}}$ arbitrarily large, otherwise the estimate would hold after adjusting $C_{1}$. Let $C_{i}$ be a series of constants with $C_{i} \rightarrow \infty$. Then there is a sequence $s_{i}$ of sections of $S$ with $\operatorname{Tr}\left(s_{i}\right)=0$ such that

$$
\begin{gathered}
\left\|s_{i}\right\|_{L^{1}} \rightarrow \infty, \\
\left\|s_{i}\right\|_{L^{1}} \geq C_{i} M\left(K s^{s_{i}}\right) .
\end{gathered}
$$

Set $l_{i}=\left\|s_{i}\right\|_{L^{1}}$ and $u_{i}=l_{i}^{-1} s_{i}$, so $\left\|u_{i}\right\|_{L^{1}}=1$, and $\sup \left|u_{i}\right| \leq C$. We may assume the $s_{i}$ are smooth, and preserved by the group $A$.

Lemma 5.4. After going to a subsequence, $u_{i}-u_{\infty}$ weakly in $L_{1}^{2}(S)$. The limit is nontrivial. If $\Phi: \mathbf{R} \times \mathbf{R} \rightarrow \mathbf{R}$ is a positive smooth function such that $\Phi\left(\lambda_{1}, \lambda_{2}\right)<\left(\lambda_{1}-\lambda_{2}\right)^{-1}$ whenever $\lambda_{1}>\lambda_{2}$, then

$$
\sqrt{-1} \int_{X} \operatorname{Tr}\left(u_{\infty} \Lambda F_{K}\right)+\int_{X}\left(\Phi\left(u_{\infty}\right)\left(D^{\prime \prime} u_{\infty}\right), D^{\prime \prime} u_{\infty}\right)_{K} \leq 0 .
$$

Proof. The second condition on $s_{i}$ is

$$
2 l_{i} \sqrt{-1} \int_{X} \operatorname{Tr}\left(u_{i} \Lambda F_{K}\right)+2 l_{i}^{2} \int_{X}\left(\Psi\left(l_{i} u_{i}\right)\left(D^{\prime \prime} u_{i}\right), D^{\prime \prime} u_{i}\right)_{K} \leq C_{i}^{-1} l_{i} .
$$

As $l \rightarrow \infty, l \Psi\left(l \lambda_{1}, l \lambda_{2}\right)$ increases monotonically to $\left(\lambda_{1}-\lambda_{2}\right)^{-1}$ if $\lambda_{1}>\lambda_{2}$ and $\infty$ if $\lambda_{1} \leq \lambda_{2}$. Fix a $\Phi$ as in the statement of the lemma. We may assume it is compactly supported since $\sup \left|u_{i}\right|$ are bounded, so for $l \gg 0$

$$
\Phi\left(\lambda_{1}, \lambda_{2}\right)<l \Psi\left(l \lambda_{1}, l \lambda_{2}\right) \text {. }
$$

Thus

$$
\sqrt{-1} \int_{X} \operatorname{Tr}\left(u_{i} \Lambda F_{K}\right)+\int_{X}\left(\Phi\left(u_{i}\right)\left(D^{\prime \prime} u_{i}\right), D^{\prime \prime} u_{i}\right)_{K} \leq \frac{1}{2} C_{i}^{-1}
$$

for $i \gg 0$.

Next we can show that $u_{i}$ are bounded in $L_{1}^{2}$. Since $\sup \left|u_{i}\right|$ are bounded there is a function $\Phi$ as above with $\Phi\left(u_{i}\right)=c$ for some small constant $c$. The above formula shows that $\int\left|D^{\prime \prime} u_{i}\right|_{K}^{2}$ are bounded. Choose a subsequence so that $u_{i} \rightarrow u_{\infty}$ weakly in $L_{1}^{2}$. 
To show that $u_{\infty}$ is nontrivial, note that for any relatively compact $Z \subset X$, $L_{1}^{2} \rightarrow L^{1}(Z)$ is compact, so

$$
\int_{Z}\left|u_{i}\right| \rightarrow \int_{Z}\left|u_{\infty}\right|
$$

On the other hand, $\sup _{X}\left|u_{i}\right| \leq C$ and $X$ has finite volume (Assumption 1), so for any $\varepsilon>0$

$$
\int_{X-Z}\left|u_{i}\right|<\varepsilon
$$

if $Z$ is big enough. Thus

$$
\int_{Z}\left|u_{\infty}\right| \geq 1-\varepsilon
$$

so $u_{\infty} \neq 0$.

Finally we do the estimate for $\Phi\left(u_{\infty}\right)$. The term $\int \operatorname{Tr}\left(u_{i} \Lambda F_{K}\right)$ is completely continuous in $u_{i} \in L_{1}^{2}$. Therefore we have shown that given any $\varepsilon>0$,

$$
\sqrt{-1} \int_{X} \operatorname{Tr}\left(u_{\infty} \Lambda F_{K}\right)+\left\|\Phi^{1 / 2}\left(u_{i}\right)\left(D^{\prime \prime} u_{i}\right)\right\|_{L^{2}}^{2} \leq \varepsilon
$$

for $i \gg 0$. Now $u_{i} \rightarrow u_{\infty}$ in $L_{0, b}^{2}$, so we can apply $4.1(\mathrm{~b})$ to conclude that $\Phi^{1 / 2}\left(u_{i}\right) \rightarrow \Phi^{1 / 2}\left(u_{\infty}\right)$ in $\operatorname{Hom}\left(L^{2}, L^{q}\right)$ for any $q<2 . D^{\prime \prime} u_{i}$ are bounded in $L^{2}$ so

$$
\sqrt{-1} \int_{X} \operatorname{Tr}\left(u_{\infty} \Lambda F_{K}\right)+\left\|F^{1 / 2}\left(u_{\infty}\right)\left(D^{\prime \prime} u_{i}\right)\right\|_{L^{q}}^{2} \leq 2 \varepsilon
$$

for $i \gg 0$. On the other hand, $\Phi^{1 / 2}\left(u_{\infty}\right)\left(D^{\prime \prime} u_{i}\right)-\Phi^{1 / 2}\left(u_{\infty}\right)\left(D^{\prime \prime} u_{\infty}\right)$ weakly in $L^{q}$. By the Hahn-Banach theorem this implies that

$$
\sqrt{-1} \int_{X} \operatorname{Tr}\left(u_{\infty} \Lambda F_{K}\right)+\left\|F^{1 / 2}\left(u_{\infty}\right)\left(D^{\prime \prime} u_{\infty}\right)\right\|_{L^{4}}^{2} \leq 2 \varepsilon .
$$

This works for any $\varepsilon>0$ and any $q<2$. If a measurable function satisfies an $L^{q}$ norm inequality which is uniform for $q<2$ then it satisfies the inequality for $q=2$. This implies the inequality in the lemma.

Lemma 5.5. The eigenvalues of $u_{\infty}$ are constant, in other words there are $\lambda_{1}$, $\ldots, \lambda_{r}$ which are the eigenvalues of $u_{\infty}(x)$ for almost all $x \in X$. The $\lambda_{i}$ are not all equal.

Proof. It suffices to show that for all smooth functions $\varphi: \mathbf{R} \rightarrow \mathbf{R}, \operatorname{Tr} \varphi\left(u_{\infty}\right)$ is a constant. We will estimate

$$
\bar{\partial} \operatorname{Tr} \varphi\left(u_{\infty}\right)=\operatorname{Tr} D^{\prime \prime} \varphi\left(u_{\infty}\right)=\operatorname{Tr} d \varphi\left(u_{\infty}\right)\left(D^{\prime \prime} u_{\infty}\right) .
$$

Suppose $N>0$ is a large number. Choose $\Phi: \mathbf{R} \times \mathbf{R} \rightarrow \mathbf{R}$ such that

$$
\Phi(\lambda, \lambda)=d \varphi(\lambda, \lambda)
$$

and

$$
N \Phi^{2}\left(\lambda_{1}, \lambda_{2}\right)<\left(\lambda_{1}-\lambda_{2}\right)^{-1}
$$


for $\lambda_{1}<\lambda_{2}$. Then

$$
\operatorname{Tr} d \varphi\left(u_{\infty}\right)\left(D^{\prime \prime} u_{\infty}\right)=\operatorname{Tr} \Phi\left(u_{\infty}\right)\left(D^{\prime \prime} u_{\infty}\right)
$$

and by Lemma 5.4

$$
\int_{X}\left|\Phi\left(u_{\infty}\right)\left(D^{\prime \prime} u_{\infty}\right)\right|_{K}^{2} \leq \frac{-\sqrt{-1}}{N} \int_{X} \operatorname{Tr}\left(u_{\infty} \Lambda F_{K}\right) .
$$

Combining these,

$$
\left\|\bar{\partial} \operatorname{Tr} \varphi\left(u_{\infty}\right)\right\|_{L^{2}}^{2} \leq \frac{C}{N} .
$$

This works for all $N$, so $\bar{\partial} \operatorname{Tr} \varphi\left(u_{\infty}\right)=0 . \operatorname{Tr} \varphi\left(u_{\infty}\right)$ is real-valued, so it is a constant. The eigenvalues are not all the same because $\operatorname{Tr}\left(u_{\infty}\right)=0$ but $u_{\infty} \neq 0$.

A consequence of this lemma is that $\varphi\left(u_{\infty}\right)$ and $\Phi\left(u_{\infty}\right)$ depend only on $\varphi\left(\lambda_{i}\right)$ and $\Phi\left(\lambda_{i}, \lambda_{j}\right), 1 \leq i, j \leq r$. Lemma 5.4 now implies that $D^{\prime \prime}\left(u_{\infty}\right)$ takes vectors with large eigenvalues to vectors with smaller ones:

Lemma 5.6. If $\Phi: \mathbf{R} \times \mathbf{R} \rightarrow \mathbf{R}$ satisfies $\Phi\left(\lambda_{i}, \lambda_{j}\right)=0$ whenever $\lambda_{i}>\lambda_{j}$, $1 \leq i, j \leq r$, then $\Phi\left(u_{\infty}\right)\left(D^{\prime \prime} u_{\infty}\right)=0$.

Proof. By the above comment, $\Phi$ can be replaced by $\Phi_{1}$ with

$$
\begin{gathered}
\Phi_{1}\left(\lambda_{i}, \lambda_{j}\right)=\Phi\left(\lambda_{i}, \lambda_{j}\right) \\
N\left(\Phi_{1}\right)^{2}\left(y_{1}, y_{2}\right)<\left(y_{1}-y_{2}\right)^{-1} \text { for } y_{1}>y_{2} .
\end{gathered}
$$

Then as before, $\left\|\Phi_{1}\left(u_{\infty}\right)\left(D^{\prime \prime} u_{\infty}\right)\right\|_{L^{2}}^{2} \leq \frac{1}{N} C$, and this works for all $N$ so $\Phi\left(u_{\infty}\right)\left(D^{\prime \prime} u_{\infty}\right)=0$. This trick is essentially the same as the argument mentioned in the appendix to $\S 4$ of [30].

The notion of weak subbundle of a holomorphic vector bundle was introduced in [30], and we can make a similar definition. Let $E$ be a Higgs bundle with a background metric $K$. An $L_{1}^{2}$-subbundle of $E$ is a section $\pi \in L_{1}^{2}(S)$ such that $\pi^{2}=\pi$ and $(1-\pi) D^{\prime \prime}(\pi)=0$.

The limiting $u_{\infty}$ constructed in the previous section gives rise to a flag of $L_{1}^{2}$-subbundles. Let $\lambda_{1}, \ldots, \lambda_{r}$ denote the eigenvalues of $u_{\infty}$ as before. Let $\{\gamma\}$ be the set of intervals between the eigenvalues and for each $\gamma$ choose $p_{\gamma}: \mathbf{R} \rightarrow \mathbf{R}$ with $p_{\gamma}\left(\lambda_{i}\right)=1$ for $\lambda_{i}<\gamma$ and $p_{\gamma}\left(\lambda_{i}\right)=0$ for $\lambda_{i}>\gamma$. Define $\pi_{\gamma}=p_{\gamma}\left(u_{\infty}\right)$.

We contend that the $\pi_{\gamma}$ are $L_{1}^{2}$ subbundles of $E$. They are in $L_{1}^{2}(S)$ by Proposition 4.1(c), and $\pi_{\gamma}^{2}=\pi_{\gamma}$ because $p_{\gamma}^{2}-p_{\gamma}$ vanishes at $\lambda_{1}, \ldots, \lambda_{r}$. To see that $\left(1-\pi_{\gamma}\right) D^{\prime \prime}\left(\pi_{\gamma}\right)=0$, recall from $\S 4$ that $D^{\prime \prime}\left(\pi_{\gamma}\right)=d p_{\gamma}\left(u_{\infty}\right)\left(D^{\prime \prime} u_{\infty}\right)$. Set $\Phi_{\gamma}\left(y_{1}, y_{2}\right)=\left(1-p_{\gamma}\right)\left(y_{2}\right) \cdot d p_{\gamma}\left(y_{1}, y_{2}\right)$. It is easy to see that $\left(1-\pi_{\gamma}\right) D^{\prime \prime}\left(\pi_{\gamma}\right)=$ $\Phi_{\gamma}\left(u_{\infty}\right)\left(D^{\prime \prime} u_{\infty}\right)$. On the other hand, $\Phi_{\gamma}\left(\lambda_{i}, \lambda_{j}\right)=0$ if $\lambda_{i}>\lambda_{j}$. By Lemma 5.6, $\Phi_{\gamma}\left(u_{\infty}\right)\left(D^{\prime \prime} u_{\infty}\right)=0$, so $\pi_{\gamma}$ is an $L_{1}^{2}$-subbundle. 
Define the degree of an $L_{1}^{2}$ subsystem $\pi$ by the Chern-Weil formula

$$
\operatorname{deg}(\pi)=\sqrt{-1} \int_{X} \operatorname{Tr}\left(\pi \Lambda F_{K}\right)-\int_{X}\left|D^{\prime \prime} \pi\right|_{K}^{2} .
$$

If $\pi$ is projection onto a subsheaf then this coincides with our previous definition.

Lemma 5.7. One of the subsystems $\pi_{\gamma}$ constructed above formally contradicts stability of $E$ in the sense that

$$
\frac{\operatorname{deg}\left(\pi_{\gamma}\right)}{\operatorname{Tr}\left(\pi_{\gamma}\right)} \geq \frac{\operatorname{deg}(E, K)}{\operatorname{rk}(E)}
$$

Proof. The trace is between 0 and $\operatorname{rk}(E)$ because $\gamma$ was an interval between two occurring eigenvalues. Write

$$
u_{\infty}=a \cdot \mathrm{Id}-\sum a_{\gamma} \pi_{\gamma} .
$$

Here $a$ is the highest eigenvalue of $u_{\infty}$ and $a_{\gamma}$ is the width of the interval $\gamma$. Consider the combination of degrees

$$
\begin{aligned}
W & =a \operatorname{deg}(E)-\sum_{\gamma} a_{\gamma} \operatorname{deg}\left(\pi_{\gamma}\right) \\
& =\sqrt{-1} \int_{X} \operatorname{Tr}\left(u_{\infty} \Lambda F\right)+\int_{X} \sum a_{\gamma}\left|D^{\prime \prime}\left(\pi_{\gamma}\right)\right|_{K}^{2} .
\end{aligned}
$$

Recall from $\S 4$ that $D^{\prime \prime}\left(\pi_{\gamma}\right)=d p_{\gamma}\left(u_{\infty}\right)\left(D^{\prime \prime} u_{\infty}\right)$, so

$$
W=\sqrt{-1} \int_{X} \operatorname{Tr}\left(u_{\infty} \Lambda F_{K}\right)+\int_{X}\left(\sum a_{\gamma}\left(d p_{\gamma}\right)^{2}\left(u_{\infty}\right)\left(D^{\prime \prime} u_{\infty}\right), D^{\prime \prime} u_{\infty}\right)_{K} .
$$

If $\lambda_{i}>\lambda_{j}, 1 \leq i, j \leq r$, then

$$
\sum a_{\gamma}\left(d p_{\gamma}\right)^{2}\left(\lambda_{i}, \lambda_{j}\right)=\left(\lambda_{i}-\lambda_{j}\right)^{-1}
$$

because $\left(d p_{\gamma}\right)^{2}\left(\lambda_{i}, \lambda_{j}\right)=\left(\lambda_{i}-\lambda_{j}\right)^{-2}$ if $\gamma$ is between $\lambda_{i}$ and $\lambda_{j}$, and is zero otherwise, and the sum of the $a_{\gamma}$ for $\gamma$ between $\lambda_{i}$ and $\lambda_{j}$ is equal to $\left|\lambda_{i}-\lambda_{j}\right|$. Now by Lemma $5.4, W \leq 0$. On the other hand

$$
\operatorname{Tr}\left(u_{\infty}\right)=a \operatorname{rk}(E)-\sum a_{\gamma} \operatorname{Tr}\left(\pi_{\gamma}\right)=0,
$$

so

$$
\frac{\operatorname{deg}\left(\pi_{\gamma}\right)}{\operatorname{Tr}\left(\pi_{\gamma}\right)} \geq \frac{\operatorname{deg}(E, K)}{\operatorname{rk}(E)}
$$

for at least one $\gamma$.

We can now quote the regularity statement for $L_{1}^{2}$-subbundles from [30]. 
Proposition 5.8. If $E$ is a Higgs bundle with background metric $K$, and if $\pi$ is an $L_{1}^{2}$-subbundle, then there is a saturated sub-Higgs-sheaf $V \subset E$ such that $\pi$ is projection onto $V$, defined where $V$ is a subbundle.

Proof. Uhlenbeck and Yau prove this when $E$ is a vector bundle [30]. Note that the statement is local, not depending on compactness of $X$. Their theorem, that a separately almost everywhere meromorphic function is meromorphic, is also proved in [28]. If $E$ is a Higgs bundle then $D^{\prime \prime}=\bar{\partial}+\theta$ so the equation $(1-\pi) D^{\prime \prime} \pi=0$ decomposes into $(1-\pi) \bar{\partial} \pi=0$ and $(1-\pi) \theta \pi=0$. If $D^{\prime \prime} \pi \in L^{2}$ then in particular $\bar{\partial} \pi \in L^{2}$. Therefore we may use the proposition for the vector bundle $E$ to conclude that $\pi$ is projection onto a saturated subsheaf $V$, and furthermore $V$ is preserved by $\theta$.

Proof of Proposition 5.3. The above constructions show that if the required estimate does not hold, then there is an $L_{1}^{2}$-subsystem $\pi$ of $E$ which formally contradicts stability of $E$. By Proposition $5.8 \pi$ is projection onto a sub-Higgs sheaf $V \subset E$. By Lemma 3.2, $\operatorname{deg}(V, K)=\operatorname{deg}(\pi)$, and by construction $V$ is preserved by the group $A$, so it contradicts stability of $E$.

\section{THE HEAT EQUATION}

In this section we will construct the solution of the nonlinear heat equation for metrics on a Higgs bundle $E$. This was done for compact $X$ and vector bundle $E$ in [7]. The proof when $E$ is a Higgs bundle is identical. We adapt the same techniques to the noncompact case. The idea is to use the exhaustion function $\phi$ given by Assumption 2, solve the heat equation with Neumann or Dirichlet boundary conditions on $X_{\varphi}$ for all time, then take the limit as $\varphi \rightarrow \infty$.

The heat equation is

$$
H^{-1} \frac{d H}{d t}=-\sqrt{-1} \Lambda F_{H}^{\perp}
$$

If we fix an initial metric $H_{0}$ and write $H_{t}=H_{0} h_{t}$ then the equation becomes

$$
\left(\frac{d}{d t}+\Delta_{0}^{\prime}\right) h=-\sqrt{-1} h \Lambda F_{0}^{\perp}+\sqrt{-1} \Lambda D^{\prime \prime}(h) h^{-1} D_{0}^{\prime}(h) .
$$

It is a nonlinear parabolic equation of type $(2,1)$ in the terminology of [13].

Lemma 6.1. Formally if $H_{t}$ is a solution of the heat equation, then

$$
\left(\frac{d}{d t}+\Delta^{\prime}\right)\left|\Lambda F_{t}^{\perp}\right|_{t}^{2} \leq 0 .
$$

Proof. For any path of metrics $H_{t}$,

$$
\frac{d}{d t} D_{H}^{\prime}=D_{H}^{\prime}\left(H^{-1} \frac{d H}{d t}\right)
$$


so

$$
\frac{d}{d t} \Lambda F=\Lambda D^{\prime \prime} D_{H}^{\prime}\left(H^{-1} \frac{d H}{d t}\right) .
$$

Now $\left|\Lambda F_{H}^{\perp}\right|_{H}^{2}=-\operatorname{Tr}\left(\Lambda F_{H}^{\perp} \Lambda F_{H}^{\perp}\right)$ so for a solution of the heat equation,

$$
\begin{aligned}
\left(\frac{d}{d t}+\Delta^{\prime}\right)\left|\Lambda F^{\perp}\right|^{2}= & -2 \operatorname{Tr}\left(\Lambda F^{\perp} \Lambda D^{\prime \prime} D^{\prime}\left(H^{-1} \frac{d H}{d t}\right)\right) \\
& -2 \sqrt{-1} \Lambda \operatorname{Tr}\left(D^{\prime \prime} \Lambda F^{\perp} D^{\prime} \Lambda F^{\perp}\right) \\
& -2 \sqrt{-1} \Lambda \operatorname{Tr}\left(\Lambda F^{\perp} D^{\prime \prime} D^{\prime} \Lambda F^{\perp}\right) \\
= & -2\left|D^{\prime \prime} \Lambda F^{\perp}\right|^{2} \\
\leq & 0
\end{aligned}
$$

(note that $\Lambda F^{\perp}$ is antiselfadjoint).

Now introduce the boundary conditions. Fix a number $\varphi$ and let $X_{\varphi}$ denote the compact space $\phi(x) \leq \varphi$, with boundary $Y_{\varphi}$. Fix a metric $K$ on $E$ over $X$, and let $\partial / \partial \nu$ denote differentiation of sections of $E$ in the direction perpendicular to the boundary using the metric connection $d_{K}$. We will consider metrics $H$ on $E$ over $X_{\varphi}$ satisfying either the Neumann boundary conditions

$$
\left.\frac{\partial}{\partial \nu} H\right|_{Y_{\varphi}}=0
$$

or the Dirichlet boundary conditions

$$
\left.H\right|_{Y_{\varphi}}=\left.K\right|_{Y_{\varphi}}
$$

Start with a metric $H_{0}$ satisfying one of these sets of conditions (for example $K$ ), and try to construct a solution $H_{t}$ of the nonlinear heat equation with the boundary conditions. Since the heat equation is parabolic, and the Neumann or Dirichlet boundary conditions are good, the solution exists for short time [13]. Suppose we know that the solution exists for $0 \leq t<T$. We will extend the solution to time $T$, and hence beyond, using the methods of [7] and [13].

Lemma 6.2. $\sup _{X_{\varphi}}\left|\Lambda F^{\perp}\right|^{2}$ is decreasing with time in a solution of the heat equation with boundary conditions.

Proof. By applying $\partial / \partial \nu$ to both sides of the heat equation, $\left|\Lambda F^{\perp}\right|^{2}$ satisfies the corresponding boundary conditions. By Lemma 6.1 and the maximum principle, its supremum is decreasing with time.

Proposition 6.3. If $H_{t}$ is a solution of the heat equation with boundary conditions defined for $0 \leq t<T$, then $H_{t}$ approach a continuous limit $H_{T}$ in $C^{0}$ norm as $T \rightarrow t$ and $H_{t}$ are bounded in $L_{2}^{p}$ uniformly in $t$, for all $p$.

Proof. Following [7] define

$$
\sigma\left(H_{1}, H_{2}\right)=\operatorname{Tr}\left(H_{1}^{-1} H_{2}\right)+\operatorname{Tr}\left(H_{2}^{-1} H_{1}\right)-2 \operatorname{rk}(E) .
$$


It gives a way of measuring distance between metrics. The same calculation as in [7, Proposition 13], using Lemma 3.1, shows that

$$
\left(\frac{d}{d t}+\Delta^{\prime}\right) \sigma\left(H_{1}, H_{2}\right) \leq 0
$$

if $H_{1}$ and $H_{2}$ are solutions of the heat equation. Furthermore, if $H_{1}$ and $H_{2}$ satisfy the boundary conditions, then so does $\sigma\left(H_{1}, H_{2}\right)$, so $\sigma$ is decreasing with time. The argument of [7, Corollary 15] shows that $H_{t} \rightarrow H_{T}$ in $C^{0}$ as $t \rightarrow T$. The proof of the proposition is completed by the following lemma.

Lemma 6.4. If $H_{t}$ is a family of metrics on $E$ over $X_{\varphi}$ with $H_{t} \rightarrow H_{T}$ in $C^{0}$, if $H_{t}$ satisfy Neumann or Dirichlet boundary conditions, and if $\sup \left|\Lambda F^{\perp}\right|$ is bounded uniformly in $t$, then $H_{t}$ are bounded in $L_{2}^{p}$ uniformly in $t$.

Proof. The same as in [7, Lemma 19], but using the boundary conditions. Fix a background metric satisfying the boundary conditions, with which to measure derivatives. First we contend that $H_{t}$ are bounded in $C^{1}$. If not then for some subsequence $t_{i}$ there are points $x_{i} \in X_{\varphi}$ with $\sup |d H|=m_{i}$ achieved at $x_{i}$, $m_{i} \rightarrow \infty$. Let $d_{i}$ denote the distance from $x_{i}$ to the boundary $Y_{\varphi}$. There are two cases. If

$$
\lim \sup d_{i} m_{i}=\varepsilon>0
$$

then we can choose balls of radius $\leq d_{i}$ around $x_{i}$ and rescale by a factor of $m_{i} / \varepsilon$ to a ball of radius 1 , so $\sup |d H|=\varepsilon$ is attained at the origin. Now the rest of the proof of [7, Lemma 19] works. On the other hand suppose

$$
\lim \sup d_{i} m_{i}=0 \text {. }
$$

We may assume $x_{i}$ approach a point $y$ on the boundary. Choose half-balls of radius $1 / m_{i}$ around $y$ and rescale by a factor of $m_{i}$ to the unit half-ball. In the rescaled picture the points $x_{i}$ still approach $y$. After rescaling, $\left|\Lambda F^{\perp}\right|$ is still bounded, $H$ is bounded, and $\sup |d H|=1$. Lemma 3.1(c) implies that $\Delta^{\prime}(H)$ is bounded, and since $H$ satisfies boundary conditions along the face of the half-ball, $H$ is bounded in $L_{2}^{p}$ on a smaller half-ball. Thus $H_{t} \rightarrow H_{\infty}$ in $C^{1}$ norm on a smaller ball. But $|d H|=1$ is attained at $x_{i} \rightarrow y$, but the total variation of $H_{t}$ goes to zero, since the original metrics approached a $C^{0}$ limit, and we rescaled by larger and larger factors. This is a contradiction so $H_{t}$ are bounded in $C^{1}$. To finish the lemma and hence the proposition, Lemma 3.1(c) together with the bounds on $H, \Lambda F^{\perp}$, and $|d H|$ show that $\Delta^{\prime}(H)$ is bounded. Elliptic estimates with boundary conditions show that $H$ is bounded in $L_{2}^{p}$.

Remark. If $H_{t}$ does not satisfy boundary conditions, then $\sup g|d H|$ is still bounded, where $g$ is the function giving distance to the boundary, for then only the first case occurs. Therefore $H_{t}$ are bounded in $L_{2, \text { loc }}^{p}$ on the interior. 
Corollary 6.5. If $H$ is a solution of the heat equation for $0 \leq t<T$ then it can be extended past $T$; so the heat equation with Neumann or Dirichlet boundary conditions has a solution defined for all time.

Proof. Since the equation is quadratic in the first derivative of $H$ we can apply Hamilton's method [13] (fractional derivatives etc.) to deduce that $H_{t} \rightarrow H_{T}$ in $C^{\infty}$, and hence by the existence, patching, and regularity theorems of [13] the solution can be continued past $T$.

Now we will use this existence result to prove the existence of a solution to the heat equation on $X$.

Proposition 6.6. Let $X$ satisfy Assumptions 1, 2, 3, and let $E$ be a Higgs bundle over $X$. Suppose $K$ is a metric satisfying the assumption that $\sup |\Lambda F| \leq B$. Then there is a unique solution $H$ to the heat equation with $\operatorname{det}(H)=\operatorname{det}(K)$, with $H_{0}=K$, and such that $\sup _{X}|H|_{K}<\infty$ on each finite interval of time. For this solution, $\sup \left|\Lambda F_{H}\right|_{H} \leq B$.

Proof. It suffices to prove existence and uniqueness for a finite interval $0 \leq$ $t \leq T$. For each $\varphi$ such that $Y_{\varphi}$ is smooth, lift the inward pointing normal vector field $\partial / \partial \nu$ to act on $E$ as before, using the metric $K$. For each $\varphi$ let $H_{\varphi, t}$ be the solution with Neumann or Dirichlet boundary conditions given by Corollary 6.5. We will take the limit as $\varphi \rightarrow \infty$, by getting a $C^{0}$ bound, then $C^{0}$ convergence, and then an $L_{2, \text { loc }}^{p}$ bound using the remark following Lemma 6.4. First notice that Lemma 6.2 is independent of $\varphi$, and the initial bound $\left|\Lambda F_{K}\right|_{K} \leq B$ is uniform on $X$, so $\left|\Lambda F_{H}^{\perp}\right|_{H} \leq B$ for all $\varphi$ and $t$. Write $H=K h$. For each $\varphi, \operatorname{Tr}\left(h_{\varphi}\right)$ satisfies the corresponding boundary conditions and

$$
\begin{aligned}
\left(\frac{d}{d t}+\Delta^{\prime}\right) \operatorname{Tr}(h) & =-\sqrt{-1} \operatorname{Tr}\left(h \Lambda F_{K}\right)+\sqrt{-1} \Lambda \operatorname{Tr}\left(D^{\prime \prime}(h) h^{-1} D_{K}^{\prime}(h)\right) \\
& \leq C_{1} \operatorname{Tr}(h)+C_{2}
\end{aligned}
$$

with $C_{1}$ and $C_{2}$ independent of $\varphi$ (Lemma 3.1(c)). The maximum principle implies that $\operatorname{Tr}(h)$, and hence $|H|_{K}$, are bounded on the finite interval $0 \leq$ $t \leq T$. The bound is independent of $\varphi$.

Lemma 6.7. Suppose $u$ is a function defined on some $X_{\varphi} \times[0, T]$, satisfying

$$
\begin{gathered}
\left(\frac{d}{d t}+\Delta^{\prime}\right) u \leq 0, \\
\left.u\right|_{t=0}=0
\end{gathered}
$$

and suppose there is a bound $\sup _{X_{\varphi}} u \leq C_{4}$. Then

$$
u(x, t) \leq \frac{C_{4}}{\varphi}\left(\phi(x)+C_{3} t\right) .
$$


Proof. The inequality holds for $t=0$ and for $x \in Y_{\varphi}$. Assumption 2 is that $|\Delta \phi| \leq C_{3}$, so

$$
\left(\frac{d}{d t}+\Delta^{\prime}\right)\left(\phi+C_{3} t\right) \geq 0 \text {. }
$$

Apply the maximum principle.

If $H$ and $J$ are two solutions of the heat equation on $X \times[0, T]$ which are both bounded with respect to the initial conditions $K$, apply the above lemma to $u=\sigma(H, J) . \quad u$ is defined on all $X_{\varphi}$ so the conclusion of the lemma becomes $u=0$, which is the uniqueness $H=J$.

Similarly to get $C^{0}$ convergence of $H_{\varphi}$ on compact sets, let $u_{\varphi \psi}=$ $\sigma\left(H_{\varphi}, H_{\psi}\right)$. The $C^{0}$ bound derived above gives the bound on $u$, and $u$ is a subsolution for the heat operator with $u(0)=0$. So if $1 \leq \tau \leq \varphi \leq \psi$ then

$$
\sigma\left(H_{\varphi}, H_{\psi}\right) \leq C \frac{\tau}{\varphi}
$$

on $X_{\tau}$. For fixed $\tau, H_{\varphi}$ is Cauchy for $\varphi \rightarrow \infty$.

Lemma 6.4 and the subsequent remark imply that on a fixed relatively compact $Z \subset X, H_{\varphi, t}$ are bounded in $L_{2}^{p}(Z)$ as $\varphi \rightarrow \infty$. The bound is uniform in $t$. Let $L_{2 / 1}^{p}(Z \times[0, T])$ denote the space of metrics with two $L^{p}$ derivatives in the space direction and one in the time direction. By the heat equation, the time derivative of $H$ is bounded in $L^{p}$ so $H_{\varphi}$ are bounded in $L_{2 / 1}^{p}$. By going to a subsequence we may assume that for each relatively compact open set, $H_{\varphi} \rightarrow H$ in $L_{2 / 1}^{p}$. By the Sobolev embedding $H_{\varphi} \rightarrow H$ in $C^{1 / 0}$ over compact sets. Therefore the limit is a solution of the heat equation. It satisfies the same $C^{0}$ bound, as well as the bound $\sup \left|\Lambda F_{H}\right|_{H} \leq B$.

Remark. If a group $A$ acts on $X$ and $E$ as in Theorem 1, and preserves $K$, then it preserves the curvature, so it preserves the heat equation. The uniqueness of the solution to the heat equation implies that the solution $H_{t}$ is preserved by $A$.

\section{Proof of Theorem 1}

Suppose $K$ is a metric on $E$ with $\sup \left|\Lambda F_{K}\right|_{K} \leq B$ and suppose that $(E, K)$ is stable (with respect to the action of a group $A$ as before). By Proposition 6.6 there is a solution $H$ of the heat equation, defined for all time, with $\operatorname{det}(H)=\operatorname{det}(K)$ and $\sup |H|_{K}<\infty$ uniformly on finite intervals of time, and $\sup \left|\Lambda F_{H}\right|_{H} \leq B$.

Lemma 7.1. $H_{t}$ is in the same component as $K$ in the space $\mathscr{P}$ of metrics, and $M\left(K, H_{t}\right)$ is continuously differentiable in $t$ with

$$
\frac{d}{d t} M\left(K, H_{t}\right)=-\int_{X}\left|\Lambda F_{H_{t}}^{\perp}\right|_{H_{t}}^{2} .
$$

Proof. First we show that $\|H\|_{P}$ is bounded. For finite time intervals $H$ is bounded and $\Lambda F_{H}$ is bounded (in $H$ norm which is equivalent to $K$ norm) 
so by Lemma 3.1(c) it suffices to show that $\int\left|D^{\prime \prime}(h) h^{-1 / 2}\right|^{2}<\infty$. It suffices to get a uniform bound over compact sets. Following the previous section find the solution $h$ as a limit of solutions $h_{\varphi}$ satisfying Neumann boundary conditions on $X_{\varphi}$. Since $h_{\varphi} \rightarrow h$ in $L_{2, \text { loc }}^{p}$ it suffices to show that

$$
\int_{X_{\varphi}}\left|D^{\prime \prime}\left(h_{\varphi}\right) h_{\varphi}^{1 / 2}\right|^{2} \leq C
$$

with $C$ independent of $\varphi$. To prove this, take the trace of Lemma 3.1(c), and integrate over $X_{\varphi}$. The bounds on $h_{\varphi}$ and $\Lambda F$ obtained in the proof of Proposition 6.6 bound one term independently of $\varphi$. The last term is the integral we want to bound, so the first part of the lemma is proved by noting that

$$
\int_{X_{\varphi}} \Delta^{\prime} \operatorname{Tr}\left(h_{\varphi}\right)=0
$$

since $\operatorname{Tr}\left(h_{\varphi}\right)$ satisfies Neumann boundary conditions.

Next we show that the derivative of $M\left(K, H_{t}\right)$ is equal to $-\left\|\Lambda F^{\perp}\right\|_{L^{2}}^{2}$. By Proposition 5.1 it suffices to do this for $t=0$. Write $H_{t}=K e^{s_{t}}$. The heat equation gives

$$
\lim _{t \rightarrow 0} \frac{s_{t}}{t}=-\sqrt{-1} \Lambda F_{K}^{\perp}
$$

uniformly on compact sets. The bound $\left|\Lambda F^{\perp}\right| \leq B$ and the heat equation imply that

$$
\sup \left|s_{t}\right| \leq C t
$$

so

$$
\lim _{t \rightarrow 0} \sqrt{-1} \frac{1}{t} \int_{X} \operatorname{Tr}\left(s \Lambda F_{K}\right)=\int_{X} \operatorname{Tr}\left(\Lambda F^{\perp} \Lambda F\right)=-\int_{X}\left|\Lambda F_{K}^{\perp}\right|^{2} .
$$

As to the second term in $M$, write $h=e^{s}$ and note that since these are bounded,

$$
\int_{X}\left(\Psi(s) D^{\prime \prime} s, D^{\prime \prime} s\right)_{K} \leq C\left\|D^{\prime \prime}(h) h^{-1 / 2}\right\|_{L^{2}}^{2}
$$

so it suffices to show that

$$
\lim _{t \rightarrow 0} \frac{1}{t}\left\|D^{\prime \prime}(h) h^{-1 / 2}\right\|_{L^{2}}^{2}=0 .
$$

On each $X_{\varphi}$ use Lemma 3.1(c):

$$
\Delta^{\prime} \operatorname{Tr}\left(h_{\varphi}\right)=\sqrt{-1} \operatorname{Tr}\left(\left(h_{\varphi}-1\right)\left(\Lambda F_{H_{\varphi}}-\Lambda F_{K}\right)\right)-\left|D^{\prime \prime}\left(h_{\varphi}\right) h_{\varphi}^{-1 / 2}\right|^{2} .
$$

Integrating the laplacian yields zero because of the Neumann boundary conditions, and $\sup \left|h_{\varphi, t}-1\right| \leq C t$ independent of $\varphi$ because of the heat equation and the bound for $\Lambda F$. For a relatively compact $Z \subset X_{\varphi} \subset X$,

$$
\int_{Z}\left|D^{\prime \prime}\left(h_{\varphi}\right) h_{\varphi}^{-1 / 2}\right|^{2} \leq C t \int_{X_{\varphi}}\left|\Lambda F_{H_{\varphi}}-\Lambda F_{K}\right|
$$


As $\varphi \rightarrow \infty, h_{\varphi} \rightarrow h$ in $L_{1}^{2}(Z)$ and then we can let $Z \rightarrow X$ so

$$
\left\|D^{\prime \prime}\left(h_{t}\right) h_{t}^{-1 / 2}\right\|_{L^{2}}^{2} \leq C t\left\|\Lambda F_{H_{t}}-\Lambda F_{K}\right\|_{L^{1}} .
$$

On the other hand, $\Lambda F$ is continuous in $t$, uniformly on compact sets, and it is bounded, so

$$
\lim _{t \rightarrow 0}\left\|\Lambda F_{H_{t}}-\Lambda F_{K}\right\|_{L^{1}}=0
$$

Proof of Theorem 1. Assume $(E, K)$ is stable with respect to the action of a group $A$. Let $H_{t}=K h_{t}$ be the solution of the heat equation constructed in the last section. It is $A$-invariant, so the estimate of Proposition 5.3 applies. Thus $\sup \left|H_{t}\right|_{K} \leq C$ independent of $t<\infty$ since $M$ is decreasing by Lemma 7.1. Furthermore $M\left(K, H_{t}\right)$ is bounded below, so there is a subsequence of times $t_{i} \rightarrow \infty$ such that $\left\|\Lambda F_{i}^{\perp}\right\|_{L^{2}}^{2} \rightarrow 0$. Next the formula for $M$ shows that

$$
\int_{X}\left|D^{\prime \prime}\left(h_{t}\right)\right|^{2} \leq C
$$

independent of $t$, so we may further restrict to a subsequence so that $H_{i} \rightarrow H_{\infty}$ weakly in $L_{1}^{2}$. In particular $H_{i} \rightarrow H_{\infty}$ in $L^{2}$ on any relatively compact open set, but $H_{i}$ are bounded uniformly on $X$ so $H_{i}$ are Cauchy in $L^{2}(X)$. By the uniform bound for $|\Lambda F|$ and Lemma 3.1(d) we have

$$
\Delta \log \operatorname{Tr}\left(H_{i}^{-1} H_{j}\right) \leq 2 B
$$

so Assumption 3 implies that $\log \operatorname{Tr}\left(H_{i}^{-1} H_{j}\right) \rightarrow 0$ in $C^{0}$, in other words $H_{i} \rightarrow$ $H_{\infty}$ in $C^{0}$. The remark following Lemma 6.4 implies that $H_{i}$ are bounded in $L_{2 \text {,loc }}^{p}$ so by going to a subsequence, $H_{i} \rightarrow H_{\infty}$ weakly in $L_{2, \text { loc }}^{p}$. Thus $F_{H_{\infty}}$ is defined, and $\Lambda F_{H_{\infty}}^{\perp}=0$. Elliptic regularity and the equation of Lemma 3.1(c) imply that $H_{\infty}$ is smooth. It is mutually bounded with respect to $K$, preserved by the group $A$, and $D^{\prime \prime}\left(K^{-1} H\right) \in L^{2}$.

We turn now to the proof of Proposition 3.5. Suppose $\nu$ is a $(1,1)$ current on $X$ with $\partial \nu=\bar{\partial} \nu=0$, and say $\nu$ is compactly supported. Then define

$$
M_{\nu}\left(K, K e^{s}\right)=\sqrt{-1} \int_{X} \operatorname{Tr}\left(s F_{K}\right) \nu \omega^{n-2}-\sqrt{-1} \int_{X} \operatorname{Tr}\left(\Psi(s)\left(D^{\prime \prime} s\right) D^{\prime} s\right) \nu \omega^{n-2} .
$$

Lemma 7.2. Suppose $f$ is a compactly supported function on $X$, and set $\nu=$ $-2 \sqrt{-1} \partial \bar{\partial} f$. Then

$$
M_{\nu}(K, H)=\int_{X} f\left(\operatorname{Tr}\left(F_{K} \wedge F_{K}\right)-\operatorname{Tr}\left(F_{H} \wedge F_{H}\right)\right) \omega^{n-2} .
$$

Proof. Differentiate both sides with respect to $H$. Proposition 5.1 holds for the functional $M_{\nu}$ (the proof is the same), so on the left side if $K$ is fixed and $H$ varied,

$$
\delta M_{\nu}(K, H)=\sqrt{-1} \int_{X} \operatorname{Tr}\left(H^{-1} \delta H F_{H}\right) \nu \omega^{n-2} .
$$


On the right side the curvature varies according to

$$
\delta F_{H}=D^{\prime \prime} D_{H}^{\prime}\left(H^{-1} \delta H\right) .
$$

The fact that $\theta^{2}=0$ implies $\left(D^{\prime \prime}\right)^{2}=0$ and $\left(D_{H}^{\prime}\right)^{2}=0$ so we have the Bianchi identities

$$
D^{\prime \prime} F_{H}=D_{H}^{\prime} F_{H}=0 \text {. }
$$

Therefore

$$
\begin{aligned}
\int_{X} f \delta \operatorname{Tr}\left(F_{H} \wedge F_{H}\right) \omega^{n-2} & =2 \int_{X} f \bar{\partial} \partial \operatorname{Tr}\left(H^{-1} \delta H F_{H}\right) \omega^{n-2} \\
& =-\sqrt{-1} \int_{X} \nu \operatorname{Tr}\left(H^{-1} \delta H F_{H}\right) \omega^{n-2}
\end{aligned}
$$

since $f$ is compactly supported. This formula is the adjunction formula used by Donaldson in [7].

Fix the assumption that $0 \leq \sqrt{-1} \partial \bar{\partial} \phi \leq C \omega$. Recall that $X_{t}$ is the set of $x$ with $\phi(x) \leq t$ and $Y_{t}$ is its boundary. Set

$$
\begin{gathered}
f_{t}(x)=\max \left(0,1-\frac{1}{t} \phi(x)\right), \\
\nu_{t}=-2 \sqrt{-1} \partial \bar{\partial} f_{t} .
\end{gathered}
$$

Write

$$
\nu_{t}=\nu_{t}^{+}+\nu_{t}^{-}
$$

with

$$
\nu_{t}^{+}=\left.\frac{2}{t} \sqrt{-1} \partial \bar{\partial} \phi\right|_{X_{t}}
$$

and $\nu_{t}^{-}$a negative current concentrated on $Y_{t}$.

Lemma 7.3. With the above notation for a fixed $t$, suppose that $K$ and $H=K e^{s}$ are metrics with $\left.H\right|_{Y_{t}}=\left.K\right|_{Y_{t}}$. Then

$$
\int f_{t} \operatorname{Tr}\left(F_{H} \wedge F_{H}\right) \omega^{n-2} \leq \int f_{t} \operatorname{Tr}\left(F_{K} \wedge F_{K}\right) \omega^{n-2}+\frac{C}{t} \int_{X_{t}}|s|\left|F_{K}\right| .
$$

Proof. Note that $\left.s\right|_{Y_{t}}=0$ and $\nu_{t}^{-}$is concentrated on $Y_{t}$ so

$$
\frac{d}{d y} M_{\nu_{t}}\left(K, K e^{y s}\right)=\sqrt{-1} \int \operatorname{Tr}\left(s F_{K e^{y s}}\right) \nu_{t}^{+} \omega^{n-2}
$$

and

$$
\begin{aligned}
\frac{d^{2}}{d y^{2}} M_{\nu_{t}}\left(K, K e^{y s}\right)= & \sqrt{-1} \int \operatorname{Tr}\left(s D^{\prime \prime} D_{K e^{y s}}^{\prime} s\right) \nu_{t}^{+} \omega^{n-2} \\
= & -\sqrt{-1} \int \operatorname{Tr}\left(D^{\prime \prime} s D_{K e^{y s}}^{\prime} s\right) \nu_{t}^{+} \omega^{n-2} \\
& -\sqrt{-1} \int \operatorname{Tr}\left(s D_{K e^{y s}}^{\prime} s\right) \bar{\partial}\left(\nu_{t}^{+}\right) \omega^{n-2}
\end{aligned}
$$


The last term is zero because $\bar{\partial}\left(\nu_{t}^{+}\right)$is a current of order one concentrated along $Y_{t}$. The other term is positive since $\nu_{t}^{+} \geq 0$. The derivative of $M$ at $y=0$ is $\sqrt{-1} \int \operatorname{Tr}\left(s F_{K}\right) \nu_{t}^{+} \omega^{n-2}$. Integrating from $y=0$ to $y=1$ gives

$$
M_{\nu_{t}}\left(K, K e^{s}\right) \geq-C \int_{X_{t}}|s|\left|F_{K}\right| .
$$

Now apply Lemma 7.2.

Proof of Proposition 3.5. Suppose $H=K h$ is the solution to the heat equation starting with a metric $K$. Find $h$ as a limit of solutions $h_{\varphi}$ satisfying Dirichlet boundary conditions on $X_{\varphi}$. Fix a finite interval of time so $\left|h_{\varphi}\right| \leq C$ uniformly in $\varphi$. Note that $h_{\varphi} \rightarrow h$ in $C^{\infty}$ uniformly on compact sets. Suppose $Z \subset X$ is a compact set. By Lemma 7.3,

$$
\int_{X} f_{\varphi} \operatorname{Tr}\left(F_{H_{\varphi}} \wedge F_{H_{\varphi}}\right) \omega^{n-2} \leq \int_{X} f_{\varphi} \operatorname{Tr}\left(F_{K} \wedge F_{K}\right) \omega^{n-2}+\frac{C}{\varphi} \int\left|F_{K}\right| .
$$

$\Lambda F_{K}$ is bounded so if the quantity on the right in Proposition 3.5 is not $\infty$ then $\int\left|F_{K}\right|<\infty$. Note that $f_{\varphi} \rightarrow 1$ as $\varphi \rightarrow \infty$. By the Riemann bilinear relations (cf. 3.4)

$$
\operatorname{Tr}(F \wedge F) \omega^{n-2} \geq-C|\Lambda F|^{2} \omega^{n},
$$

and $\sup \left|\Lambda F_{H_{\varphi}}\right| \leq C$ uniformly in $\varphi$ (6.2), so

$$
\int_{X-Z} f_{\varphi} \operatorname{Tr}\left(F_{H_{\varphi}} \wedge F_{H_{\varphi}}\right) \omega^{n-2} \geq-C \operatorname{vol}(X-Z) .
$$

Therefore

$$
\int_{Z} f_{\varphi} \operatorname{Tr}\left(F_{H_{\varphi}} \wedge F_{H_{\varphi}}\right) \omega^{n-2} \leq \int_{X} f_{\varphi} \operatorname{Tr}\left(F_{K} \wedge F_{K}\right) \omega^{n-2}+\frac{C}{\varphi}+C \operatorname{vol}(X-Z) .
$$

The constants do not depend on $\varphi$ or $Z$. Take the limit as $\varphi \rightarrow \infty$ :

$$
\int_{Z} \operatorname{Tr}\left(F_{H} \wedge F_{H}\right) \omega^{n-2} \leq \int_{X} \operatorname{Tr}\left(F_{K} \wedge F_{K}\right) \omega^{n-2}+C \operatorname{vol}(X-Z)
$$

then take the limit as $Z \rightarrow X$.

Remark. If the initial metric we started with satisfies $\sup \left|F_{K}\right|<\infty$ then equality holds in Proposition 3.5. To see this use the following lemma.

Lemma 7.4. Suppose $H$ and $K$ are mutually bounded metrics such that $\sup _{X}\left|F_{K}\right|<\infty$ and $\int_{X}\left|D^{\prime \prime} K^{-1} H\right|^{2}<\infty$. Then

$$
\int_{X} \operatorname{Tr}\left(F_{K} \wedge F_{K}\right) \omega^{n-2} \leq \int_{X} \operatorname{Tr}\left(F_{H} \wedge F_{H}\right) \omega^{n-2} .
$$

Proof. The total mass of the current $\nu_{t}$ is bounded by $C / t$ for some $C$, and $F_{K}$ and $s=\log K^{-1} H$ are bounded, so the first term in $M_{\nu_{t}}$ approaches zero. On the other hand, $\nu_{t} \leq C \omega / t$ so the second term in $M_{\nu_{t}}$ is almost negative:

$$
-\sqrt{-1} \int_{X} \operatorname{Tr}\left(\Psi(s)\left(D^{\prime \prime} s\right) \wedge D^{\prime} s\right) \wedge \nu_{t} \wedge \omega^{n-2} \leq \frac{C}{t} \int_{X}\left|D^{\prime \prime} s\right|^{2}
$$

Now apply Lemma 7.2 and take the limit as $t \rightarrow \infty$. 


\section{Systems of Hodge bundles and variations of Hodge StRucture}

A complex variation of Hodge structure $([10,11,5])$ over $X$ is a $C^{\infty}$ vector bundle $V$ with a decomposition $V=\bigoplus_{p+q=w} V^{p, q}$ and a flat connection $D$ satisfying the Griffiths transversality condition

$$
D: V^{p, q} \rightarrow A^{0,1}\left(V^{p+1, q-1}\right) \oplus A^{1}\left(V^{p, q}\right) \oplus A^{1,0}\left(V^{p-1, q+1}\right)
$$

and such that a polarization exists; this is a sesquilinear form $\langle\cdot, \cdot\rangle$ on $V$, hermitian symmetric or antisymmetric as $w$ is even or odd, invariant under $D$, such that the Hodge decomposition is orthogonal, and such that $(\sqrt{-1})^{p-q}\langle\cdot, \cdot\rangle$ $>0$ on $V^{p, q}$.

A system of Hodge bundles on $X$ is a direct sum of holomorphic bundles $E=\bigoplus_{p, q} E^{p, q}$ together with maps $\theta: E^{p, q} \rightarrow E^{p-1, q+1} \otimes \Omega_{X}^{1}$ such that $\theta^{2}: E \rightarrow E \otimes \Omega_{X}^{2}$ is zero. In particular a system of Hodge bundles is a Higgs bundle with action of the group $A=U(1) \times U(1)$. A metric on a system of Hodge bundles means a metric on the Higgs bundle $E$, preserved by $A$. It is therefore a direct sum of metrics on the bundles $E^{p, q}$, and we can look at the indefinite hermitian form

$$
\langle u, v\rangle_{K}=(\sqrt{-1})^{p-q}(u, v)_{K}
$$

for $u, v \in E^{p, q}$. Given a metric $K$ we get a metric connection $D_{K}$ and this preserves the indefinite form. It also satisfies the Griffiths transversality condition. Thus if the curvature of $K$ is zero, we get a variation of Hodge structure. Conversely given a variation of Hodge structure, we get a system of Hodge bundles in a natural way, and a polarization defines a flat metric on the system of Hodge bundles. The operators $\bar{\partial}$ and $\theta$ are obtained as components of the flat connection of the variation, and the integrability conditions $\bar{\partial}^{2}=0$, $\bar{\partial}(\theta)=0$, and $\theta^{2}=0$ are obtained from $D^{2}=0$. Therefore a system of Hodge bundles with a flat metric is the same thing as a polarized complex variation of Hodge structure.

Suppose $E$ is a system of Hodge bundles. A subsystem of Hodge sheaves is a sub-Higgs sheaf of $E$ which is preserved by the group $A$. Make the same definitions of degree and stability as in $\S 3$. Thus if $X$ is not compact, these depend on the choice of an initial metric $K$ on $E$ such that $\sup \left|\Lambda F_{K}\right|<\infty$. Theorem 1 in this case says that if $E$ is a system of Hodge bundles which is stable with respect to an intial metric $K$, then there is a comparable metric $H$ with the same determinant, such that $\Lambda F_{H}^{\perp}=0$.

Proposition 8.1. Suppose $X$ is compact with Kähler metric $\omega$. The category of complex variations of Hodge structure is equivalent to the full subcategory of systems of Hodge bundles $E$ such that $c_{1}(E)=0, c_{2}(E) \cdot[\omega]^{n-2}=0$, and $E$ is a direct sum of stable systems of Hodge bundles of degree zero. 
Proof. $X$ satisfies the assumptions of $\S 2$. If $E$ is stable of degree zero, then by usual harmonic theory we may choose a metric on $\operatorname{det}(E)$ with $\Lambda F=0$. By applying Theorem 1 we get a metric on $E$ with $\Lambda F=0$. If $E$ is a direct sum of systems of degree zero then do this on each summand. Since $\theta^{2}=0$ we may apply Proposition 3.4 to conclude that $F=0$, so $E$ comes from a variation of Hodge structure. On the other hand if $E$ comes from a variation of Hodge structure then by Proposition 3.3 it is a direct sum of stable systems of degree zero, and all the Chern classes vanish since it has a flat metric. If $E^{\prime}$ and $E^{\prime \prime}$ are variations of Hodge structure, the morphisms $E^{\prime} \rightarrow E^{\prime \prime}$ in the category of variations are the maps which preserve the connection. If $f: E^{\prime} \rightarrow E^{\prime \prime}$ is a map of systems of Hodge bundles, we have to prove that $D(f)=0$. Let $K$ be the flat metric on $E^{\prime} \oplus E^{\prime \prime}$ and let $H$ be the metric obtained by pulling back with the automorphism $1+f$ of $E^{\prime} \oplus E^{\prime \prime}$. Thus $H=K h$ with $h=(1+f)(1+f)^{*}$ and $H$ is also flat. Lemma 3.1 implies that

$$
\Delta^{\prime} \operatorname{Tr}(h)=-\left|D^{\prime \prime}(h) h^{-1 / 2}\right|^{2}
$$

but $\int_{X} \Delta^{\prime} \operatorname{Tr}(h)=0$ so $D^{\prime \prime}(h)=0$. Therefore $D^{\prime \prime}(f)=0$ and $D^{\prime \prime}\left(f^{*}\right)=$ $D^{\prime}(f)^{*}=0$ as needed.

Remark. Corollary 3.6 gives a construction of variations of Hodge structure when $X$ in noncompact. This leads to the somewhat complicated problems of choosing initial metrics and understanding stability. We will discuss these when $X$ is a curve, in $\S 10$. The higher dimensional case will be left to a later time.

We now discuss a principal bundle version of Proposition 8.1. Define a Hodge group to be a semisimple real algebraic Lie group $G_{0}$ together with a Hodge decomposition of the complexified Lie algebra

$$
\mathfrak{g}=\bigoplus_{p} \mathfrak{g}^{p,-p}
$$

such that $\left[\mathfrak{g}^{p,-p}, \mathfrak{g}^{r,-r}\right] \subset \mathfrak{g}^{p+r,-p-r}$ and such that $(-1)^{p+1} \operatorname{Tr}(\operatorname{ad} U \operatorname{ad} \bar{V})>0$ for $U, V \in \mathfrak{g}^{p,-p}$. If $G_{0}$ is a Hodge group, let $K_{0}$ be the subgroup corresponding to Lie algebra $\mathfrak{k}=\mathfrak{g}_{0}^{0,0}$. It is the subgroup of elements $k$ such that $\operatorname{ad}(k)$ preserves the Hodge decomposition of $\mathfrak{g}$. In particular $\operatorname{ad}(k)$ preserves the above positive definite form so $K_{0}$ is compact. Let $G, K$ denote the complexifications of $G_{0}, K_{0}$ respectively.

Define a principal system of Hodge bundles to be a principal holomorphic $K$ bundle $P$ together with a holomorphic map

$$
\theta: T(X) \rightarrow P \times_{K} \mathfrak{g}^{-1,1}
$$

such that $[\theta(u), \theta(v)]=0$. Here $T(X)$ is the holomorphic tangent bundle. A metric $H$ for a principal system of Hodge bundles is a $C^{\infty}$ reduction of structure group of $P$ from $K$ to $K_{0}$, in other words a principal $K_{0}$ bundle $P_{H} \subset P$. If $(P, \theta)$ is a system of Hodge bundles with metric $P_{H} \subset P$, there is a unique connection $d_{H}$ on $P_{H}$ which is compatible with the holomorphic 
structure of $P$. Let $\bar{\theta}_{H}$ be the complex conjugate of $\theta$. Then $D_{H}=d_{H}+\theta+\bar{\theta}_{H}$ is a connection on the principal $G_{H}$-bundle $R_{H}=P_{H} \times G_{0}$. A principal variation of Hodge structure for Hodge group $G_{0}$ is a principal system of Hodge bundles $P$ together with a metric $P_{H}$ such that the curvature of the associated connection $D_{H}$ is zero.

Suppose $\left(P_{H}, D\right)$ is a principal variation of Hodge structure. Fix a universal cover $\tilde{X}$. The flat connection on $R_{H}$ means that we can choose a flat trivialization $\varphi:\left.R_{H}\right|_{\widetilde{X}} \cong G_{0}$. Then for each $x \in \widetilde{X}$ there is a right $K_{0}$-coset $\varphi P_{H}(x) \subset G_{0}$. Thus we get a map $\widetilde{X} \rightarrow G_{0} / K_{0}$. Just as in the case of usual variations of Hodge structure, $G_{0} / K_{0}$ is a homogeneous complex manifold with horizontal tangent subbundle, and the map $\tilde{X} \rightarrow G_{0} / K_{0}$ is holomorphic and horizontal. $\pi_{1}(X)$ is the group of covering transformations of $\tilde{X}$. The covering transformations act on $\left.R_{H}\right|_{\widetilde{X}}$ but will not preserve the trivialization $\varphi$. Thus there is a representation $\sigma: \pi_{1}(X) \rightarrow G_{0}$ such that $\sigma(\gamma) \varphi(r)=\varphi(\gamma r)$ for $\left.r \in R_{H}\right|_{\widetilde{X}}$ and $\gamma \in \pi_{1}(X)$. The map $x \mapsto \varphi P_{H}(x)$ from $\widetilde{X}$ to $G_{0} / K_{0}$ is equivariant under this representation:

$$
\varphi P_{H}(\gamma x)=\varphi \gamma P_{H}(x)=\sigma(\gamma) \varphi P_{H}(x) .
$$

The horizontal holomorphic tangent space to $G_{0} / K_{0}$ at $\varphi P_{H}(x)$ is identified with $P \times_{K} \mathfrak{g}^{-1,1}(x)$ and the differential of $\tilde{X} \rightarrow G_{0} / K_{0}$ is the map $\theta: T(X) \rightarrow$ $P \times_{K} \mathfrak{g}^{-1,1}$.

A Hodge representation of $G_{0}$ is a complex representation $V$ of $G_{0}$ together with Hodge decomposition $V=\bigoplus V^{p, q}$ such that the action of $\mathfrak{g}$ is compatible with Hodge type, and such that $K_{0}$ preserves Hodge type (this may not be automatic if $K_{0}$ is not connected). A polarization for $V$ is a metric $K$ for the Hodge structure such that the associated indefinite Hermitian form

$$
\langle u, v\rangle_{K}=(\sqrt{-1})^{p-q}(u, v)_{K}, \quad u, v \in V^{p, q}
$$

is $G_{0}$-invariant. In particular $\mathfrak{g}$ is a polarized Hodge representation. If $V$ is a Hodge representation of $G$ and $P$ is a principal system of Hodge bundles then $P \times_{K} V$ becomes a system of Hodge bundles. A polarization for $V$ and a metric $P_{H}$ for $P$ together give a metric $H$ on the system of Hodge bundles.

Suppose $V$ is an irreducible polarized Hodge representation of $G_{0}$. We say that a principal system of Hodge bundles $(P, \theta)$ is $V$-stable if the system of Hodge bundles $P \times_{K} V$ is stable. Note that since $G_{0}$ is semisimple, $\operatorname{det}\left(P \times_{K} V\right)=1$. If $X$ is noncompact then this notion depends on the choice of an initial metric such that $\Lambda F$ is bounded.

Proposition 8.2. If a principal system of Hodge bundles $(P, \theta)$ is $V_{i}$-stable for some family of irreducible polarized Hodge representations $\left\{V_{i}\right\}$ which faithfully represents $\mathfrak{g}$, then there exists a metric $P_{H}$ such that the curvature of the metric connection is primitive:

$$
\Lambda F=0 .
$$


If in addition $X$ is compact and $c_{2}\left(P \times_{K} \mathfrak{g}\right) \cdot[\omega]^{n-2}=0$ then $F=0$ so this gives a principal variation of Hodge structure.

Proof. Fix an initial metric $P_{H_{0}}$. If $V$ is any faithful polarized Hodge representation of $G_{0}$ then this gives an initial metric $H_{0}$ on $V$. The solution of the heat equation

$$
h^{-1} \frac{d h}{d t}=-\sqrt{-1} \Lambda F
$$

for metrics $H_{t}=H_{0} h_{t}$ on $V$ is unique and compatible with direct sums and tensor products. Thus if $w$ is a tensor preserved by $G$ then $h_{t}(w)=1$ for all $t$. This implies that $h_{t} \in G$ [6], so the family of metrics comes from a family of metrics $P_{H_{t}}$ on $P$. Now stability of the $V_{i}$ implies that the heat equation converges to a metric with $\Lambda F=0$. If $X$ is compact and $c_{2}\left(P \times_{K} \mathfrak{g}\right) \cdot[\omega]^{n-2}=0$ then we can apply Proposition 3.4 to conclude that $F=0$.

Remark. If $X$ is not compact but $0 \leq \sqrt{-1} \partial \bar{\partial} \phi \leq C \omega$ and we have a good initial metric with $c_{2}\left(P \times_{K} \mathfrak{g}\right) \cdot[\omega]^{n-2}=0$ then we can apply Corollary 3.6 to conclude that $F=0$.

Finally we discuss a special case, projective variations of Hodge structure. Let $V=\bigoplus V^{p, q}$ be a Hodge structure with a metric $J$ and associated indefinite form $\langle\cdot, \cdot\rangle_{J}$. Set

$$
G_{0}=\operatorname{Aut}\left(V,\langle\cdot, \cdot\rangle_{J}\right) / U(1) .
$$

Its Lie algebra $\mathfrak{g}$ is the space $\operatorname{End}(V)^{\perp}$ of endomorphisms of $V$ of trace zero; it has a natural Hodge decomposition, making $G_{0}$ into a Hodge group. The compact isotropy subgroup is $K_{0}=\operatorname{Aut}\left(\left\{V^{p, q}\right\}, J\right) / U(1)$. A projective variation of Hodge structure modeled on $V$ is a principal variation $\left(P_{H}, D\right)$ for the Hodge group $G_{0}$.

Lemma 8.3. Suppose $X$ is compact. If a system of Hodge bundles $E$ is a direct sum of stable systems of the same slope (i.e. degree divided by rank) and satisfies equality in Proposition 3.4, then there is a corresponding projective variation of Hodge structure. The differential of the classifying map is given by the map $\theta: T(X) \rightarrow(\text { End } E)^{-1,1}$.

Proof. $E$ gives rise to a principal system of Hodge bundles, in other words a principal $K$-bundle $P$. By Theorem 1 and Proposition 3.4 there is a metric $H$ on $E$ with $F^{\perp}=0$. This gives a metric $P_{H}$ on $P$ with zero curvature, hence a projective variation.

Corollary 8.4. Suppose $L$ is a line bundle on $X$ with a nonzero map $L \rightarrow \Omega_{X}^{1}$, and with $L . H^{n-1}>0$. Then $L^{2} \cdot H^{n-2} \leq 0$ and if equality holds, there is a nontrivial representation $\pi_{1}(X) \rightarrow P S L_{2}(\mathbf{R})$ and an equivariant map from $\tilde{X}$ to the upper half-plane.

Proof. The system of Hodge bundles $\left(L, \mathscr{O}_{X}\right)$ is stable by the condition $L . H^{n-1}>0$. The Bogomolov-Gieseker inequality 3.4 is $L^{2} \cdot H^{n-2} \leq 0$. If 
equality holds then we get a projective variation of Hodge structure. The Hodge group corresponding to $\left(L, \mathscr{O}_{X}\right)$ is $\operatorname{PSU}(1,1)$ and the classifying space is the unit disc, equal to the upper half plane via $P S U(1,1) \cong P S L_{2}(\mathbf{R})$.

\section{UNIFORMIZATION}

Algebro-geometric characterizations of when a quasiprojective variety is uniformized by a Hermitian symmetric space of the noncompact type have been obtained by Yau and others using Kähler-Einstein metrics [17, 18, 31]. In this section we will treat the question of uniformization from the point of view of variations of Hodge structure, applying the results of the previous sections. We will assume that $X$ is compact because then the conditions obtained above for existence of variations of Hodge structure are algebraic in nature.

A Hodge group of Hermitian type is a Hodge group such that the Hodge decomposition of $\mathfrak{g}$ has only types $(1,-1),(0,0),(-1,1)$, and such that $G_{0}$ has no compact factors. In this case $K_{0} \subset G_{0}$ is a maximal compact subgroup and $\mathscr{D}=G_{0} / K_{0}$ is a Hermitian symmetric space of the noncompact type, in other words a bounded symmetric domain. Furthermore all bounded symmetric domains arise this way. One should be careful that there may be several groups $G_{0}$ corresponding to the same $\mathscr{D}$, but their connected components are all isogenous, so the Lie algebra $\mathfrak{g}$ and its Hodge decomposition are determined.

Fix a Hodge group $G_{0}$ of Hermitian type. A uniformizing bundle is a principal system of Hodge bundles $(P, \theta)$ such that $\theta: T(X) \rightarrow P \times_{K} g^{-1,1}$ is an isomorphism. This data is just the data of a holomorphic reduction of structure group for $T(X)$ to $K \rightarrow G l(n)$, where the map $K \rightarrow G l(n)$ is given by the representation $\mathfrak{g}^{-1,1}$ of $K$. A uniformizing variation of Hodge structure is a uniformizing bundle $P$ together with a flat metric.

Proposition 9.1. Let $X$ be compact and let $\tilde{X}$ be the universal cover of $X$. Then $\widetilde{X}$ is isomorphic to the bounded symmetric domain $\mathscr{D}$ if and only if a uniformizing variation of Hodge structure exists for some Hodge group $G_{0}$ with $\mathscr{D}=G_{0} / K_{0}$.

Proof. Suppose a uniformizing variation exists. Then the classifying map is a holomorphic $\pi_{1}(X)$-equivariant map $\widetilde{X} \rightarrow \mathscr{D}$, with differential given by $\theta$ : $T(X) \stackrel{\cong}{\rightrightarrows} P \times_{K} \mathfrak{g}^{-1,1}$. In particular it is a local diffeomorphism. Now $\pi_{1}(X)$ acts freely and discretely on $\widetilde{X}$ with compact quotient $X$, and preserves a metric on $\mathscr{D}$. An easy argument shows that in this situation, $\widetilde{X} \rightarrow \mathscr{D}$ must be a covering. But $\mathscr{D}$ is simply connected so $\widetilde{X} \cong \mathscr{D}$ as desired. Conversely suppose we are given $\tilde{X} \cong \mathscr{D}$. Let $G_{0}=\operatorname{Aut}(\mathscr{D})$ be the group of holomorphic automorphisms. This is a Hodge group with $G_{0} / K_{0}=\mathscr{D}$. Since $\pi_{1}(X)$ acts holomorphically on $\tilde{X} \cong \mathscr{D}$ we get a representation $\pi_{1}(X) \rightarrow G_{0}$ and the equivariant map $\widetilde{X} \rightarrow \mathscr{D}$ gives a principal variation of Hodge structure by inverting the procedure of $\xi 8$. It is uniformizing because the differential is an isomorphism. 
Remark. If $X$ is not compact then we must include the additional assumption that there exists a complete metric on $X$ such that the norm of $\theta^{-1}$ is bounded measured in this metric. If there is a uniformizing variation satisfying this assumption then the map $\widetilde{X} \rightarrow \mathscr{D}$ must be a covering so $X$ is uniformized.

Write the decomposition of $\mathfrak{g}$ into simple ideals $\mathfrak{g}=\bigoplus \mathfrak{g}_{i}$. These are subHodge structures, and if $G_{0}$ is connected they are Hodge representations of $G_{0}$. If $(P, \theta)$ is a uniformizing bundle, we can decompose $P \times_{K} \mathfrak{g}=\bigoplus P \times_{K} \mathfrak{g}_{i}$ locally on $X$, and the $P \times_{K} \mathfrak{g}_{i}$ are locally subsystems of Hodge bundles. There is a unique finest global decomposition $P \times_{K} \mathfrak{g}=\bigoplus U_{j}$ such that $U_{j}$ are locally direct sums of the simple ideals $P \times_{K} \mathfrak{g}_{i}$. The $U_{j}$ may not come from representations of $G$. However, there is a subgroup $G^{\prime} \subset G$ which has the same connected component as $G$, such that the structure group of $P$ can be reduced to $K^{\prime}=K \cap G^{\prime}$, and such that each $U_{j}$ comes from a representation $\mathfrak{u}_{j}$ of $G_{0}^{\prime}$. The $\mathfrak{u}_{j}$ will be direct sums of the $\mathfrak{g}_{i}$. From now on we will always assume that such a reduction has been made.

Say that a uniformizing bundle $(P, \theta)$ is stable if it is $\mathfrak{u}_{j}$-stable for all $j$, in other words if the systems $U_{j}$ are stable. To understand this condition requires a lemma about Lie algebras of the type we are considering. W. Schmid explained to me how to prove it.

Lemma 9.2. Suppose $\mathfrak{g}_{0}$ is the Lie algebra of a Hodge group of Hermitian type. Suppose $\mathfrak{g}$ is simple. If $e \in \mathfrak{g}^{1,-1}$ then $\left[\mathfrak{g}^{-1,1},\left[\mathfrak{g}^{-1,1}, e\right]\right]=\mathfrak{g}^{-1,1}$.

Proof. Let

$$
\begin{aligned}
\mathfrak{k}_{0} & =\mathfrak{g}_{0}^{0,0}, \\
\mathfrak{p}_{0} & =\left(\mathfrak{g}^{1,-1} \oplus \mathfrak{g}^{-1,1}\right) \cap \mathfrak{g}_{0}
\end{aligned}
$$

so $\mathfrak{g}_{0}=\mathfrak{k}_{0} \oplus \mathfrak{p}_{0}$ is a Cartan decomposition. To prove the lemma it suffices to show that if $e, f \in \mathfrak{g}^{1,-1}$ then there exist $x, y \in \mathfrak{g}^{-1,1}$ such that

$$
B([e, x],[f, y]) \neq 0 \text {. }
$$

Here $B(u, v)$ denotes the Killing form $-\operatorname{Tr}(\operatorname{ad} u \cdot \operatorname{ad} v)$. Suppose $[e, \bar{f}] \neq 0$. Set $x=\bar{f}, y=\bar{e}$, so $[f, y]=-\overline{[e, x]}$. But $B$ is definite on $\mathfrak{k}_{0}$ so the associated Hermitian form is definite on $\mathfrak{k}$. Thus $B([e, x],[f, y]) \neq 0$.

So we may assume $[e, \bar{f}]=0$. Then $e+\bar{e}$ and $f+\bar{f}$. lie in a maximal abelian subalgebra $\mathfrak{a}_{0} \subset \mathfrak{p}_{0}$. We may choose a Cartan subalgebra $\mathfrak{h} \subset \mathfrak{k}$ and roots $\beta_{1}, \ldots, \beta_{r}$ for $\mathfrak{g}^{1,-1}$ such that $\mathfrak{a}_{0}$ is the span of the elements $E_{\beta_{i}}+\overline{E_{\beta_{i}}}$ where $E_{\beta}$ is the standard vector of weight $\beta$. In this situation the $\beta_{i}$ are strongly orthogonal: $\beta_{i} \perp \beta_{j}$ and in fact $\beta_{i}+\beta_{j}$ and $\beta_{i}-\beta_{j}$ are not roots. We may write

$$
e=\sum a_{i} E_{\beta_{i}}, \quad f=\sum b_{j} E_{\beta_{j}} .
$$

The condition that $[e, \bar{f}]=0$ translates to: for all $i$ either $a_{i}=0$ or $b_{i}=0$. Choose $i$ with $a_{i} \neq 0$ and $j$ with $b_{j} \neq 0$. The results of Moore [23] give a 
root $\alpha$ of $\mathfrak{k}$ such that the projection of $\alpha$ onto the space spanned by the root vectors $\beta_{1}, \ldots, \beta_{r}$ is

$$
\frac{1}{2}\left(\beta_{i}-\beta_{j}\right) .
$$

In particular $\left(\alpha, \beta_{i}\right)>0$ so $\alpha-\beta_{i}$ is a root, and similarly $-\alpha-\beta_{j}$ is a root. Set

$$
x=E_{\alpha-\beta_{i}}, \quad y=E_{-\alpha-\beta_{j}} .
$$

Note that $\left(\alpha-\beta_{i}, \beta_{l}\right)=0$ for $l \neq i, j$. Thus $\alpha-\beta_{i}+\beta_{l}$ is not a root because $\alpha-\beta_{i}-\beta_{l}$ is not a root, and similarly $-\alpha-\beta_{j}+\beta_{l}$ is not a root. Therefore

$$
\begin{gathered}
{[e, x]=\left[E_{\beta_{i}}, E_{\alpha-\beta_{i}}\right]=E_{\alpha},} \\
{[f, y]=\left[E_{\beta_{j}}, E_{-\alpha-\beta_{j}}\right]=E_{-\alpha}}
\end{gathered}
$$

up to scalar multiples, so $B([e, x],[f, y]) \neq 0$.

Corollary 9.3. Suppose $\mathfrak{g}$ is the Lie algebra of a Hodge group of Hermitian type. If $W \subset \mathfrak{g}$ is a sub-Hodge structure such that $\left[\mathfrak{g}^{-1,1}, W\right] \subset W$ then

$$
\operatorname{dim} W^{-1,1} \geq \operatorname{dim} W^{1,-1}
$$

and if equality holds then $W$ is a direct sum of ideals of $\mathfrak{g}$.

Corollary 9.4. Suppose $(P, \theta)$ is a uniformizing system of Hodge bundles. Then the following conditions are equivalent:

(i) $(P, \theta)$ is stable.

(ii) $P \times_{K} \mathfrak{g}$ is a direct sum of stable systems of degree zero.

(iii) Each saturated subsystem of Hodge sheaves $W \subset P \times_{K} \mathfrak{g}$ with $\operatorname{deg}(W) \geq$ 0 is locally a direct sum of simple ideals $P \times_{K} \mathfrak{g}_{i}$.

Proof. Write $P \times_{K} \mathfrak{g}=\bigoplus U_{j}$ as above, then (i) $\Rightarrow$ (ii) is clear. For (ii) $\Rightarrow$ (iii): $\operatorname{deg}(W)=0$, and by Proposition 3.3 if $W \subset P \times_{K} \mathfrak{g}$ is saturated then it is a direct summand, say $P \times \mathfrak{g}=W \oplus W_{1}$. In particular $W$ and $W_{1}$ are subsystems of Hodge bundles. Corollary 9.3 applies to $W$ and $W_{1}$, and in fact equality must hold for both because it holds for their direct sum, so $W$ is locally a direct sum of simple ideals. For (iii) $\Rightarrow$ (i): if $W \subset U_{j}$ is a saturated subsystem with $\operatorname{deg}(W) \geq 0$ then $W$ is locally a direct sum of simple ideals so $W=U_{j}$ by minimality of the decomposition.

Theorem 2. Suppose $X$ is compact. $\widetilde{X} \cong \mathscr{D}$ if and only if there is a uniformizing system of Hodge bundles $(P, \theta)$ for a Hodge group $G_{0}$ of Hermitian type corresponding to $\mathscr{D}$, such that $(P, \theta)$ is stable and $c_{2}\left(P \times_{K} \mathfrak{g}\right) \cdot[\omega]^{n-2}=0$.

Proof. If such a uniformizing system of Hodge bundles exists then Proposition 8.2 gives a uniformizing variation of Hodge structure $(P, D)$; Proposition 9.1 shows that $\widetilde{X} \cong \mathscr{D}$. Conversely if $\widetilde{X} \cong \mathscr{D}$, we get a uniformizing variation $(P, D)$, and the associated system of Hodge bundles has a flat metric, so 
$c_{2}\left(P \times_{K} \mathfrak{g}\right)=0$. Proposition 3.3 shows that $P \times_{K} \mathfrak{g}$ is a direct sum of stable systems of degree zero.

Corollary 9.5. Let $\mathscr{D}$ be a bounded symmetric domain, and let $X$ be a projective algebraic variety over $\mathbf{C}$. If $\mathscr{D}$ is the universal cover of $X$ then for any $\sigma \in$ $\operatorname{Aut}(\mathbf{C} / \mathbf{Q}), \mathscr{D}$ is the universal cover of $X^{\sigma}$.

This statement was already known by a combination of Margulis' theorem that $X$ is arithmetic if the rank is bigger than one, Kazhdan's result that conjugates of arithmetic varieties are arithmetic [16], and Yau's criterion in the rank one case [31]. Yau informs me that using Kähler-Einstein metrics he gets a characterization of varieties uniformized by Hermitian symmetric spaces in terms of ampleness of the canonical bundle and existence of a section of a symmetric power of the cotangent bundle.

Proposition 9.6. Suppose $X$ is projective and suppose $(P, \theta)$ is a uniformizing variation which we think of as a reduction of structure group for $T(X)$. Write $T(X)=\oplus V_{i}$ corresponding to the irreducible components of the representation $K \subset G l(n)$. If the $V_{i}$ are semistable vector bundles on $X$ of degrees strictly less that zero, then $(P, \theta)$ is stable. So if $c_{2}\left(P \times_{K} \mathfrak{g}\right) \cdot[\omega]^{n-2}=0$ then $(P, \theta)$ uniformizes $X$.

Proof. First suppose $G$ is connected. Let $\mathfrak{g}=\bigoplus \mathfrak{g}_{i}$ be the decomposition into simple ideals. Then $\mathfrak{g}^{-1,1}=\bigoplus \mathfrak{g}_{i}^{-1,1}$ is the decomposition into irreducible representations of $K$. The $P \times_{K} \mathfrak{g}_{i}^{-1,1}$ and their duals $P \times_{K} \mathfrak{g}_{i}^{1,-1}$ are semistable. Lie bracket

$$
\mathfrak{g}_{i}^{1,-1} \otimes \mathfrak{g}_{i}^{-1,1} \rightarrow \mathfrak{g}_{i}^{0,0}
$$

is surjective, the left-hand side gives a semistable vector bundle of degree zero, and the right side gives a vector bundle of degree zero. Therefore $P \times_{K}$ $\mathfrak{g}_{i}^{0,0}$ is semistable. Suppose $W \subset P \times_{K} \mathfrak{g}_{i}$ is a subsystem of Hodge sheaves. Then $\operatorname{deg}\left(W^{0,0}\right) \leq 0$ and $\operatorname{deg}\left(W^{1,-1}\right) \leq-\operatorname{rk}\left(W^{1,-1}\right) \cdot \mu$ and $\operatorname{deg}\left(W^{-1,1}\right) \leq$ $\operatorname{rk}\left(W^{-1,1}\right) \cdot \mu$ where

$$
\mu=\frac{\operatorname{deg}\left(P \times_{K} \mathfrak{g}_{i}^{-1,1}\right)}{\operatorname{rk}\left(\mathfrak{g}_{i}^{-1,1}\right)}<0 .
$$

Therefore

$$
\frac{\operatorname{deg}(W)}{\operatorname{rk}(W)} \leq\left(\operatorname{rk}\left(W^{-1,1}\right)-\operatorname{rk}\left(W^{1,-1}\right)\right) \cdot \mu \leq 0
$$

and if equality holds then $W=P \times_{K} \mathfrak{g}_{i}$, using Corollary 9.3. This proves that $P \times{ }_{K} \mathfrak{g}_{i}$ is stable, which proves the proposition when $G$ is connected.

Now suppose $G$ is not connected. Let $G^{\prime}$ be the connected component, $K^{\prime}=K \cap G^{\prime}$, and let $f: X^{\prime} \rightarrow X$ be the finite etale covering over which the structure group of $P$ can be reduced to $K^{\prime}$. The pull-backs $f^{*} V_{i}$ are semistable. If we decompose $T X^{\prime}=\bigoplus V_{k}^{\prime}$ into irreducible components for $K^{\prime}$ then the $V_{k}^{\prime}$ are direct summands of the $f^{*} V_{i}$ so they are semistable, of 
degrees $<0$. By the corollary with connected $G^{\prime}$, the pull-back $f^{*} P \times_{K} \mathfrak{g}$ is polystable. If $W \subset P \times_{K} \mathfrak{g}$ is a saturated subsystem with $\operatorname{deg}(W) \geq 0$, then $f^{*} W$ is a direct summand in $f^{*} P \times_{K} \mathfrak{g}$. By $9.3, f^{*} W$ is locally a direct sum of simple ideals, so the same is true of $W$ on $X$. Now apply 9.4 .

Corollary 9.7. Suppose $X$ is compact. If $T(X)=L_{1} \oplus \cdots \oplus L_{n}$ is a direct sum of line bundles of degrees $c_{1}\left(L_{i}\right) \cdot[\omega]^{n-1}<0$, and if $\left(c_{1}(X)^{2}-2 c_{2}(X)\right) \cdot[\omega]^{n-2}=0$, then $\tilde{X}$ is a product of $n$ copies of the unit disc.

Proof. $P$ is the principal $K=\mathbf{C}^{*} \times \cdots \times \mathbf{C}^{*}$-bundle corresponding to $L_{1} \oplus$ $\cdots \oplus L_{n}$. The class $c_{2}\left(P \times_{K} \mathfrak{g}\right)$ is represented by $F_{1} \wedge F_{1}+\cdots+F_{n} \wedge F_{n}$ where $F_{i} \in A^{2}(X)$ is the curvature of $L_{i}$, so

$$
c_{2}\left(P \times_{K} \mathfrak{g}\right)=c_{1}\left(L_{1}\right)^{2}+\cdots+c_{1}\left(L_{n}\right)^{2}=c_{1}(X)^{2}-2 c_{2}(X)
$$

up to a constant. Now apply 9.6. In this example $c_{2}\left(P \times_{K} \mathfrak{g}\right)$ was a linear combination of $c_{1}(X)^{2}$ and $c_{2}(X)$. This does not seem to be true for general $G$, but W. Schmid informs me that it is true if $K$ modulo its center is simple.

Finally we treat the unit ball. There is a canonical system of Hodge bundles $W=\left(\Omega_{X}^{1}, \mathscr{O}_{X}\right)$ on $X: W^{1,0}=\Omega_{X}^{1}$ and $W^{0,1}=\mathscr{O}_{X}$.

Proposition 9.8. Suppose $X$ is compact. If $W$ is a stable system of Hodge bundles over $X$ then

$$
\left(2 c_{2}(X)-\frac{n}{n+1} c_{1}(X)^{2}\right) \cdot[\omega]^{n-2} \geq 0
$$

and if equality holds then $X$ is uniformized by the unit ball.

Proof. Apply Lemma 8.3 to $W$. If equality holds it gives a projective variation of Hodge structure with group $P U(n, 1)$. The symmetric space corresponding to this group is the unit ball $B^{n} \subset \mathbf{C}^{n}$. The differential of the classifying map is $\theta: T(X) \cong \operatorname{Hom}\left(\Omega_{X}^{1}, \mathscr{O}_{X}\right)$ so the classifying map gives the uniformization.

If $X$ is a curve there is no Chern condition, and the only saturated subsystem of Hodge sheaves of $W=\left(\Omega_{X}^{1}, \mathscr{O}_{X}\right)$ is $\mathscr{O}_{X}$ itself. Thus $W$ is stable if and only if the genus of $X$ is $\geq 2$. Hence $X$ is uniformized by the unit disc if and only if the genus is $\geq 2$. This is the classical uniformization theorem for curves.

Remark. If $X=\bar{X}-Y$ is a noncompact curve, then one looks at $W=$ $\left(\Omega_{X}^{1}\langle\log Y\rangle, \mathscr{O}_{X}\right)$. Following $\S 10$ below, one can choose an initial metric with logarithmic growth and bounded curvature, and then $W$ is stable if and only if $\operatorname{deg} \Omega_{X}^{1}\langle\log Y\rangle>0$. Theorem 1 then gives a metric with $F^{\perp}=0$, and the uniformization theorem for $X$ follows by using the remark after Proposition 9.1 . 
Proposition 9.9 ([31, 22]). Suppose $X$ is a projective surface. If $c_{1}(X)^{2} \geq$ $3 c_{2}(X), c_{1}(X)^{2}>0$ and $c_{1}(X) \cdot[\omega]<0$ then $W$ is stable so $c_{1}(X)^{2}=3 c_{2}(X)$ and $\widetilde{X} \cong B^{2}$.

Proof. We will prove that the dual $W^{*}=\left(\mathscr{O}_{X}, T(X)\right)$ is stable. The only saturated subsystem of Hodge sheaves of rank two is $T(X)$ itself, and that is taken care of by $c_{1}(X) .[\omega]<0$. The saturated subsystems of rank one are the invertible subsheaves $L \subset T(X)$ which are subbundles outside of a finite collection of points. We have to show that $c_{1}(L) .[\omega]<\frac{1}{3} c_{1}(X) \cdot[\omega]$. Suppose the contrary. Let $M=T(X) / L$; it is torsion-free but not a line bundle. Let $N=M^{* *}$ so $N$ is a line bundle and $M \subset N$ has quotient concentrated on a finite set. $\left(\mathscr{O}_{X}, N\right)$ is a system of Hodge sheaves, and

$$
c_{1}(N) \cdot[\omega] \leq \frac{2}{3} c_{1}(X) \cdot[\omega]<0,
$$

so $\left(\mathscr{O}_{X}, N\right)$ is stable. The Bogomolov-Gieseker inequality 3.4 implies

$$
c_{1}(N)^{2} \leq 0 \text {. }
$$

$T(X)$ is equal to $N+L-(N / M)$ in the Grothendieck group, so

$$
\begin{aligned}
c_{2}(X) & =c_{1}(N) c_{1}(X)-c_{1}(N)^{2}-c_{2}(N / M) \\
& \geq c_{1}(N) c_{1}(X)-c_{1}(N)^{2}
\end{aligned}
$$

because $c_{2}(N / M) \leq 0$ since $N / M$ is concentrated on a finite set. Therefore

$$
\left(3 c_{1}(N)-2 c_{1}(X)\right)^{2} \geq 4\left(c_{1}(X)^{2}-3 c_{2}(X)\right) \geq 0
$$

by our hypothesis. On the other hand, we apply the Hodge index theorem. $2 c_{1}(X)$ is in the interior of the negative half of the cone $A^{2}>0$, while $3 c_{1}(N)$ is outside the cone and below $2 c_{1}(X)$. Therefore $3 c_{1}(N)-2 c_{1}(X)$ is strictly outside the cone, so $\left(3 c_{1}(N)-2 c_{1}(X)\right)^{2}<0$. This contradiction shows that $W$ is stable, so the uniformization $\widetilde{X} \cong B^{2}$ is attained.

This argument is essentially the same as that given by Miyaoka to show that $c_{1}^{2} \leq 3 c_{2}$ for surfaces of general type [21]. The result on uniformization is known by work of Yau and Miyaoka [31, 22]. Unfortunately our argument to prove stablity does not seem to work in higher dimensions. I do not know how to go between the condition we have given, of stability of $W$, and the condition of Yau [31], that $K_{X}$ is ample, by algebraic geometry.

\section{QuASIPRojective CURVES}

In this section we will treat the one-dimensional noncompact case. Let $X$ be a quasiprojective curve, and let $\bar{X}$ be its smooth completion. Let $\omega$ be a metric on $X$ which extends smoothly over $\bar{X}$. We will classify metrics with $L^{p}$ curvature, $p>1$, on systems of Hodge bundles over $X$, up to mutual boundedness. This together with Theorem 1 will provide a classification of 
complex variations of Hodge structure on $X$. We follow the point of view of Griffiths' article [11] using the idea of the Kashiwara-Malgrange filtration [15, 20].

Let $j: X \rightarrow \bar{X}$ be the inclusion and let $Y=\bar{X}-X$. A regular system of Hodge bundles on $X^{\mathrm{alg}}$ is an algebraic system of Hodge bundles $E$ on $X$, together with a filtration

$$
j_{*}^{\mathrm{alg}} E=\bigcup_{\alpha \in \mathbf{R}^{\gamma}} E_{\alpha}
$$

by coherent subsheaves indexed by the partially ordered set $\mathbf{R}^{Y}$, compatible with the Hodge decomposition, such that

$$
\theta: E_{\alpha}^{p, q} \rightarrow E_{\alpha}^{p-1, q+1} \otimes \Omega_{X}^{1}\langle\log Y\rangle ;
$$

such that

$$
E_{\alpha} \subset E_{\beta}
$$

whenever $\alpha_{y} \geq \beta_{y}$ for all $y \in Y$, and

$$
E_{\alpha}=\bigcap_{\beta<\alpha} E_{\beta} ;
$$

and such that

$$
E_{\alpha+\delta^{r}}=E_{\alpha}(-y)
$$

where $\delta_{z}^{y}=1$ for $y=z$ and 0 otherwise.

Construction. Suppose $E$ is an analytic system of Hodge bundles on $X$, and suppose $K$ is a metric. Then we can construct a family of sheaves $a E$ on $X$ : $a E_{\alpha}^{p, q}$ is the subsheaf of $j_{*}^{a n} E^{p, q}$ consisting of sections $e$ with growth near $y \in Y$ bounded by

$$
|e|_{K} \leq C r^{\alpha \alpha_{y}+\varepsilon}
$$

for any $\varepsilon>0$. Note that $\left.a E\right|_{X} \cong E$ canonically. The following theorem plays the role of Schmid's Nilpotent Orbit and $S L_{2}$-orbit Theorems [27].

Theorem 3. Suppose $E$ is a system of Hodge bundles on $X$ with a metric $K$, such that

$$
\int_{X}\left|F_{K}\right|^{p}<\infty
$$

for some $p>1$. Then the $a E_{\alpha,}$ are coherent, and

$$
\theta: a E_{\alpha}^{p, q} \rightarrow a E_{\alpha}^{p-1, q+1} \otimes \Omega_{X}^{1}\langle\log Y\rangle,
$$

so $a E$ is a regular system of Hodge bundles on $X^{\mathrm{alg}}$. The construction ' $a$ ' commutes with taking determinants, duals, tensor products, and first Chern class. If $H$ and $K$ are two metrics on $E$ such that $a_{H} E=a_{K} E$ then $H$ and $K$ are mutually bounded. The construction ' $a$ ' is an equivalence between the category 
of metrized systems of Hodge bundles on $X$ with $L^{p}$ curvature, with morphisms the bounded maps, and the category of regular systems of Hodge bundles on $X^{\mathrm{alg}}$.

Proof. For flat metrics $K$ this follows from [27]. We give an alternative argument which works for curved metrics. This will occupy most of the rest of the section. The theorem is a local statement near the punctures, so assume that $X$ is the punctured disc, and $\bar{X}$ is the disc. The proof of the first statement is based on the distance decreasing property for variations of Hodge structure, which works in the curved case as pointed out by Beilinson and Deligne:

Lemma 10.1. Let $E$ be a system of Hodge bundles on $X$, and suppose $p>1$. For any metric $K$ with $\int_{X}\left|F_{K}\right|^{p}<\infty$,

$$
\sup |\theta| \leq \frac{C}{|z \log | z||}
$$

where $z$ is a local coordinate around a puncture $y \in Y . C$ depends on the $L^{p}$ norm of the curvature.

Proof. Use the euclidean metric on the disc. The curvature of the unitary bundle $(\text { End } V)^{-1,1} \otimes \Omega_{X}^{1}$ is then just $d_{K}^{2}$ where $d_{K}$ is the metric connection for the holomorphic bundle $(E, \bar{\partial})$. The following statement holds away from the zero set of $\theta$ :

$$
\Delta \log |\theta|^{2} \leq \frac{\left(2 \sqrt{-1} \operatorname{ad} \Lambda d_{K}^{2}(\theta), \theta\right)}{|\theta|^{2}} .
$$

It is the statement that curvature decreases in subbundles, applied to the line subbundle of $(\text { End } V)^{-1,1} \otimes \Omega_{X}^{1}$ given by the holomorphic section $\theta$.

Now note that $d_{K}^{2}=F_{K}-\theta \bar{\theta}-\bar{\theta} \theta$. The calculation of the negative horizontal sectional curvature of the classifying space for Hodge structures [12] amounts to the inequality

$$
\frac{(2 \sqrt{-1} \operatorname{ad} \Lambda(\theta \bar{\theta}+\bar{\theta} \theta) \theta, \theta)}{|\theta|^{2}} \geq C|\theta|^{2}
$$

for some positive constant $C$. Furthermore, by multiplying the metric by a scalar factor $e^{s}$ with $s$ bounded, we may assume that the curvature $\sqrt{-1} \Lambda F_{K}$ is negative (see below). Therefore

$$
\Delta \log |\theta|^{2} \leq-C|\theta|^{2} .
$$

This holds in a distributional sense everywhere since $\log |\theta|^{2} \rightarrow-\infty$ at the zeros of $\theta$. Now applying Ahlfors' lemma [1], we are done.

This lemma allows us to conclude that $\theta: a E \rightarrow a E \otimes \Omega_{X}^{1}\langle\log Y\rangle$ preserves the filtrations. Furthermore, the curvatures of the individual bundles are bounded

$$
\left|d_{K}^{2}\right| \leq f+\frac{C}{|z \log | z||^{2}}
$$


with $f \in L^{p}$. This implies that the $a E_{\alpha}$ are coherent, by the theory of Cornalba-Griffiths [4] with a minor modification. Their main trick is to multiply the metric $K$ by a scalar factor $e^{s}$. The curvature changes by $\partial \bar{\partial} s$. If we set $s=\sigma+C \log \log |z|$ with $\Delta \sigma=f$ then this makes the curvature negative, in other words all of the eigenvalues of $\sqrt{-1} \Lambda F$ are negative. The additional term $\sigma$ does not appear in [4] but it is bounded by the $L^{p}$ estimates for $\Delta$. The rest of the argument of [4] works.

The main step will be to show that $\operatorname{det}(a E) \cong a(\operatorname{det} E)$. It is clear that $\operatorname{det}(a E)_{\alpha} \subset a(\operatorname{det} E)_{\alpha}$. The following lemma gives a preliminary bound in the other direction.

Lemma 10.2. Suppose $K$ is a metric on a constant holomorphic bundle $\mathscr{O}_{X}^{k}$ over the punctured disc, with negative curvature. Suppose that the eigenvalues of $K$ are $\leq 1$ and that

$$
|\operatorname{det}(K)| \leq C|z| .
$$

Then there is a constant section $e \in \mathbf{C}^{k}$ such that

$$
|e|_{K} \leq C|z|^{1 / 6 k} \text {. }
$$

Proof. Since curvature decreases in subbundles, for any constant section $e \in \mathbf{C}^{k}$ we have

$$
\sqrt{-1} \bar{\partial} \partial \log |e|_{K} \leq 0 \text {. }
$$

We will show that there is a sequence $r_{i}$ and a sequence of vectors $e_{i}$ whose norm in $\mathbf{C}^{k}$ is 1 , such that the area in $|z| \leq r_{i}$ where $\log \left|e_{i}\right|_{K} \leq \frac{1}{2 k} \log r_{i}$ is at least $r_{i}^{3}$. Since $\log \left|e_{i}\right|_{K}$ is subharmonic and less than zero on $|z| \leq 1$, it is easy to see that we will get

$$
\log \left|e_{i}\right|_{K} \leq \frac{1}{6 k} \log |z|
$$

in $r_{i} \leq|z| \leq 1$. Now the $e_{i}$ will approach a limit $e$ and this estimate gives

$$
\log |e|_{K} \leq \frac{1}{6 k} \log |z|
$$

as desired. To choose the $e_{i}$ proceed as follows. Choose any sequence $r_{i} \rightarrow 0$. The hypotheses imply that for any $|z| \leq r_{i}$ there is at least one $e \in \mathbf{C}^{k}$ such that $|e(z)|_{K} \leq \frac{1}{2 k} \log r_{i}$. Let $S$ denote the unit sphere in $\mathbf{C}^{k}$. For each $|z| \leq r_{i}$ then the area of the set of vectors $e$ in $S$ such that

$$
|e(z)|_{K} \leq 2 r_{i}^{1 / 2 k}
$$

is at least $r_{i}^{(2 k-1) / 2 k}$, since

$$
|e+f|_{K} \leq|e|_{K}+|f|_{\mathbf{C}^{k}} \leq 2 r_{i}^{1 / 2 k}
$$

for any vector $f$ with euclidean norm less than $r_{i}^{1 / 2 k}$. Therefore the total area in $S \times\left\{|z| \leq r_{i}\right\}$ where the $K$-norm of the vector is less than $r_{i}^{1 / 2 k}$, is at least 
$r_{i}^{3}$. But the area of $S$ is finite so we can find a single vector $e_{i} \in S$ such that this holds, as desired. This proves the lemma.

Remark. The conclusion of the lemma still holds under the weaker curvature assumption

$$
\left|F_{K}\right| \leq f+\frac{C}{|z \log | z||^{2}}
$$

with $f \in L^{p}$, because we can use the trick of modifying the metric by a scalar $e^{s}$ as above, so the curvature becomes negative. Include $\varepsilon \log |z|$ in $s$ for a small $\varepsilon$ so as to preserve the assumptions $K \leq 1$ and $\operatorname{det}(K) \leq C|z|$.

To get a precise bound for $a(\operatorname{det} E)$ we use this crude estimate on finite covers of $X$ ramified over the puncture. Let $\pi: W \rightarrow X$ be the $n$-fold cover of the unit disc $z=t^{n}$ ( $z$ is the coordinate on $X$ and $t$ the coordinate on $W)$. Let $G$ be the Galois group, the group of $n$th roots of unity. If $(E, K)$ is a metrized system of Hodge bundles on $X$ then there is an obvious pull-back $\pi^{*} E$. On the other hand if $E^{\prime}$ is a regular system of Hodge bundles on $X^{\text {alg }}$ then we can define the pull-back $\pi^{*} E^{\prime}$ as follows:

$$
\pi^{*} E_{\alpha}^{\prime}=\sum_{n \beta+m \geq \alpha} t^{m} \pi^{*}\left(E_{\beta}^{\prime}\right) .
$$

Lemma 10.3. If $(E, K)$ is a metrized system of Hodge bundles on $X$ then $a\left(\pi^{*} E\right)=\pi^{*}(a E)$.

Proof. $G$ acts on $\pi^{*} E$ over its action on $W$, and this preserves the metric. The $\mathscr{O}_{W}$-module $\pi^{*} E$ decomposes into a direct sum of $\mathscr{O}_{X}$-modules

$$
\pi^{*} E=\bigoplus_{m=0}^{n-1} t^{m} E
$$

under the action of $G$. If $e$ is a section of $\pi^{*} E$ whose norm is bounded in some way then the components of $e$ in the above decomposition are bounded in the same way, since $G$ preserves the metric. Also multiplication by $t^{m}$ just shifts the filtration. Therefore

$$
a\left(\pi^{*} E\right)=\bigoplus_{m=0}^{n-1} t^{m} a(E)
$$

with filtrations shifted suitably. This proves the lemma.

Corollary 10.4. If $E$ is a system of Hodge bundles with a metric $K$ whose curvature is in $L^{p}$ then $\operatorname{det}(a E)=a(\operatorname{det} E)$.

Proof. One direction of inclusions is obvious. Now suppose $a(\operatorname{det} E) \not \subset$ $\operatorname{det}(a E)$. Consider a ramified cover $\pi$ as above with $n$ sufficiently big so that

$$
\operatorname{det}\left(a\left(\pi^{*} E\right)_{0}\right) \subset a\left(\operatorname{det}\left(\pi^{*} E\right)\right)_{2} .
$$


Choose $n$ so that the weights $\alpha$ which occur in the filtration of $a E$, when multiplied by $n$, are all just slightly bigger than various integers. In particular

$$
a\left(\pi^{*} E\right)_{1 / 2 k} \subset a\left(\pi^{*} E\right)_{1} .
$$

Apply Lemma 10.2 to the bundle $\pi^{*} E_{0}$ to get a contradiction.

Next we contend that ' $a$ ' commutes with taking duals of line bundles. If $L$ is a line bundle with metric $K$ satisfying the assumptions, choose a nonvanishing section $l$ with polynomial growth. The curvature being in $L^{p}$ means that

$$
f=\Delta \log |l|_{K}
$$

is in $L^{p}$. The condition that the metric has polynomial growth implies that

$$
\log |l|_{K}=b \log |z|+\sigma
$$

with $\Delta \sigma=f$ weakly on $\bar{X}$, so $\sigma$ is bounded. Thus $a L_{\alpha}$ is generated by sections $z^{n} l$ such that $n+b \geq \alpha$. The metric on the dual bundle $L^{*}$ satisfies

$$
\log \left|l^{*}\right|_{K}=-b \log |z|-\sigma
$$

so $a\left(L^{*}\right)_{-\alpha}$ is generated by sections $z^{n} l^{*}$ such that $n-b \geq-\alpha$. Thus $a\left(L^{*}\right)_{-\alpha}=\left(a L_{\alpha}\right)^{*}$.

Suppose $E^{\prime}$ and $E^{\prime \prime}$ are two systems with metrics. It is immediate that the identity map extends to a morphism of regular systems on $X^{\mathrm{alg}}$ :

$$
a E^{\prime} \otimes a E^{\prime \prime} \rightarrow a\left(E^{\prime} \otimes E^{\prime \prime}\right) .
$$

Using this and the fact that the dual $E^{*}$ is a direct summand in some tensor product of copies of $E$ and $\operatorname{det}(E)^{-1}$, the identity map extends to a morphism

$$
(a E)^{*} \rightarrow a\left(E^{*}\right) \text {. }
$$

On the other hand, the pairing

$$
a\left(E^{*}\right) \otimes a E \rightarrow a\left(E^{*} \otimes E\right) \rightarrow a\left(\mathscr{O}_{X}\right)
$$

shows that the identity gives a map

$$
a\left(E^{*}\right) \rightarrow(a E)^{*} .
$$

Therefore ' $a$ ' commutes with taking duals. Similarly it commutes with taking tensor products.

Suppose $H$ and $K$ are two metrics on $E$ with $L^{p}$ curvature, such that $a_{H} E=a_{K} E$. Set

$$
\nu=\log \operatorname{Tr}\left(H^{-1} K\right)+\log \operatorname{Tr}\left(K^{-1} H\right) .
$$

Note that $\nu \geq 0$. By Lemma 3.1,

$$
\Delta \nu \leq b
$$

where $\int_{X} b^{p}<\infty$. The condition $a_{H} E=a_{K} E$ means that the identity map is a section of $a_{H \otimes K}\left(E^{*} \otimes E\right)_{0}$ and of $a_{K \otimes H}\left(E^{*} \otimes E\right)_{0}$. This implies that $\nu$ 
grows slower than $\varepsilon|\log | z||$ for any $\varepsilon$. By the proof of Proposition 2.2 and the subsequent remark, we conclude that $\Delta \nu \leq b$ weakly on $\bar{X}$. Since $b \in L^{p}, \nu$ is bounded (as in Propositions 2.1 and 2.4). Therefore $H$ and $K$ are mutually bounded.

Now we can show that the functor $a$ is fully faithful. If $E^{\prime}$ and $E^{\prime \prime}$ are systems with metrics $K$, and if $f: a E^{\prime} \rightarrow a E^{\prime \prime}$ is a morphism of regular systems on $X^{\text {alg }}$ then

$$
h=\left(\begin{array}{ll}
1 & 0 \\
f & 1
\end{array}\right)
$$

is an isomorphism of the system $E^{\prime} \oplus E^{\prime \prime}$. It preserves the structure $a\left(E^{\prime} \oplus E^{\prime \prime}\right)$ so by the above, the pull-back $K h^{*} h$ of the metric $K$ is bounded with respect to $K$, in other words $h^{*} h$ is bounded. In particular, the off-diagonal term $f$ is bounded.

To prove the last statement of the theorem, that $a$ is an equivalence, we have to show that it is essentially surjective. Suppose $E$ is a regular system on $X^{\text {alg }}$. We need to construct a metric $K$ on $\left.E\right|_{X}$ such that $a_{K}\left(\left.E\right|_{X}\right)=E$ and such that the curvature is in $L^{p}$ for some $p>1$. Proceed as in [27, Lemma 6.24]. The two essential cases are a line bundle $L$, and the system $W=\left(W^{1,0}, W^{0,1}\right)$ where the components are equal to $\mathscr{O}_{X}$ with the obvious filtration, and $\theta=d z / z$. Every line bundle is of the form $\mathscr{O}_{X}(\alpha)$ for some $\alpha$, where $1 \in \mathscr{O}_{X}(\alpha)_{\beta}$ whenever $\beta \leq \alpha$. For this system use the metric $|1|=|z|^{\alpha}$. In the system $W$ let $w^{p, q}$ be the unit section of $W^{p, q}=\mathscr{O}_{X}$. It is easy to see that the metric given by

$$
\left|w^{1,0}\right|=|\log | z||^{1 / 2}, \quad\left|w^{0,1}\right|=\left.|\log | z\right|^{-1 / 2}
$$

is flat. Using these two constructions, we get flat metrics on any systems which are direct sums of tensor or symmetric products of the above ones. To complete the construction, approximate any system by such a one, sufficiently to get a metric with $L^{p}$ curvature. For any $E$ set

$$
g r_{\alpha}(E)=E_{\alpha} / \sum_{\beta>\alpha} E_{\beta} .
$$

Choose an isomorphism of regular bundles on $X^{\text {alg }}$

$$
E \cong \bigoplus_{0 \leq \alpha<1} \operatorname{gr}_{\alpha}(E) \otimes_{\mathbf{C}} \mathscr{O}_{X}(\alpha)
$$

This will be an isomorphism of filtered bundles. Define $\operatorname{res}(d z / z)=1$ and use the above isomorphism to define a new $\theta_{1}: E \rightarrow E \otimes \Omega_{X}^{1}\langle\log Y\rangle$ :

$$
\theta_{1}=\operatorname{gr}(\operatorname{res}(\theta)) \text {. }
$$

The system $\left(E, \theta_{1}\right)$ is a direct sum of symmetric products of line bundles and systems $W$ as above (this is the Jacobson-Morosov theorem, applied as in [27]). Therefore there is a metric $H$ which is flat with respect to $\theta_{1}$ and has 
the required growth properties. Write $\theta=\theta_{1}+g$, so there is some $\varepsilon>0$ such that

$$
g: E_{\alpha} \rightarrow E_{\alpha+\varepsilon} \otimes \Omega_{X}^{1}\langle\log Y\rangle
$$

(the filtration is really indexed discretely so take $\varepsilon$ smaller than the smallest distance between consecutive steps). Therefore

$$
|g|_{H} \leq C|z|^{\varepsilon / 2-1} \text {. }
$$

The difference between the curvatures of the systems $(E, \theta)$ and $\left(E, \theta_{1}\right)$ using the same metric $H$, is

$$
\theta_{1} \bar{g}+\bar{g} \theta_{1}+g \bar{\theta}_{1}+\bar{\theta}_{1} g+g \bar{g}+\bar{g} g
$$

and the norm of this is less than $C|z|^{\varepsilon / 2-2}$. Therefore the curvature of the metric $H$ on the system $(E, \theta)$ is in $L^{p}$ for some $p>1$. This shows that the regular system $E$ comes from a metrized system over $X$.

Remark. This argument gives information on the norms of holomorphic sections of $a E$. In the flat case this information follows from Schmid's norm estimates. Thus we have given an alternative proof of the norm estimates for holomorphic sections of the Hodge bundles. I am not sure if one can derive the norm estimates for flat sections from this, for example one might still need the Nilpotent Orbit Theorem. Also Schmid's $S L_{2}$ Orbit Theorem provides more precise information on the higher order asymptotics.

The theorem is proved except for the statement about Chern classes. More generally suppose $E$ is a system over $X$ with metric $K$, and $V$ is a strict subsystem of $a E$. In other words $\left.V\right|_{X} \subset E$ is a saturated subsystem and $V_{\alpha}=V \cap a E_{\alpha}$. Then we can define the degree $\operatorname{deg}(V)$ in two different ways.

$$
\operatorname{deg}^{\text {an }}(V)=\int_{X} c_{1}(V, K)
$$

and

$$
\operatorname{deg}^{\text {alg }}(V)=\operatorname{deg}^{\text {alg }}\left(\bigwedge^{\text {top }} V\right)
$$

where the degree of a line bundle is defined by

$$
\operatorname{deg}^{\operatorname{alg}}\left(\mathscr{O}_{X}(\alpha \cdot y)\right)=2 \pi \alpha
$$

and $\operatorname{deg}^{\mathrm{alg}}(L)$ is the usual degree if $L$ comes from a line bundle over $\bar{X}$.

Lemma 10.5. If $E$ is a metrized system as in the above theorem, and $V \subset a E$ is a strict subsystem, then $\operatorname{deg}^{\text {an }}(V)=\operatorname{deg}^{\text {alg }}(V)$.

Proof. It suffices to consider the case where $V$ is a line bundle. Furthermore we may tensor with a line bundle that comes from $\bar{X}$, so we may assume $V_{0}=\mathscr{O}_{\bar{X}}$. The metric $K$ restricts to a metric on $\mathscr{O}_{X}$. Set $h=|1|_{K}$. It is a function on $X$ with growth approximately $|z|^{a_{y}}$ near $y \in Y, 0 \leq a_{y}<1$. In fact if $g$ is a function such that $g=|z|^{a_{y}}$ near $y$ then $|\log h-\log g| \leq C|\log \log | z||$ near the punctures, since the metric $K$ is bounded with respect to a metric which 
satisfies this, namely the metric on $\left.a E\right|_{X}$ constructed above (we can choose the isomorphism $E \cong \bigoplus \mathrm{gr}_{\alpha} E \otimes \mathscr{O}_{X}(\alpha)$ so that $V$ is constant). It is easy to see that

$$
\sqrt{-1} \int_{X} \bar{\partial} \partial \log g=\operatorname{deg}^{\mathrm{alg}}(V)
$$

because the integral of the distribution $\bar{\partial} \partial \log g$ over $\bar{X}$ is zero and this differs from the above integral by contributions from delta functions at the punctures. These contributions are the same as the terms that go into $\operatorname{deg}^{\text {alg }}(V)$ from the $y \in Y$. So we have to prove that

$$
\int_{X} \Delta s=0
$$

if $|s| \leq \log \log |z|$ near the punctures. We may also assume that $\Delta s \leq b$ where $b \in L^{p}$, since curvature decreases in subsystems and the curvature of $E$ is in $L^{p}$. Proposition 2.2 and the remark following it show that $\Delta s \leq b$ weakly on $\bar{X}$. A negative distribution on $\bar{X}$ which restricts to a function on $X$ restricts to an $L^{1}$ function on $X$. Therefore the integral in question exists. Apply the remark following Proposition 2.2 to show that $\Delta s=\left.\Delta s\right|_{X}$ weakly on $\bar{X}$. The integral over $\bar{X}$ of the distribution $\Delta s$ vanishes, so the integral in question also vanishes. This proves the lemma. Theorem 3 is now proved.

Lemma 10.6. Suppose $X$ is a curve with smooth completion $\bar{X}$. Let $E$ be a vector bundle with an extension $\bar{E}$ and suppose $K$ is a metric on $E$ with polynomial growth with respect to the completion $\bar{E}$. Assume $\int_{X}\left|F_{K}\right|<\infty$. If $V \subset E$ is an $L_{1}^{2}$ subbundle analytically defined over $X$, then it extends to a subbundle $\bar{V} \subset \bar{E}$.

Proof. Note first of all that nothing depends on the base metric. All statements are local so assume $X$ is a punctured disc, $\bar{X}$ is a disc, the metric $\omega$ is the euclidean metric on the disc, and $\bar{E} \cong \mathscr{O}_{X}^{r}$. Let $H$ denote the constant metric on $\bar{E}$ obtained from this identification. Polynomial growth of $K$ means that $|K|_{H}$ and $|H|_{K}$ grow slower than $|z|^{a}$ for some $a$ where $z$ is the coordinate on the disc.

By taking an exterior power and using the Plücker embedding we may assume that $V$ is a line bundle. This line bundle is trivial so choose a section $v$.

$$
f=\partial \bar{\partial} \log |v|_{K}
$$

is the curvature of $V$ with respect to the induced metric. If $\pi$ is the projection onto $V$ then the $L_{1}^{2}$ condition is that $\bar{\partial} \pi \in L^{2}$. The Chern-Weil formula for the curvature of $V$ is

$$
f=\operatorname{Tr}\left(\pi F_{K}\right)-|\bar{\partial} \pi|^{2} \omega
$$

so $\int_{X}|f|<\infty$ by our hypotheses. In fact by choosing our disc in $\bar{X}$ to be small enough in the first place, we may assume $\int|f|<2$. Now consider $f$ as a distribution on $\bar{X}$ and let $s$ be a solution of

$$
\partial \bar{\partial}(s)=f
$$


given by convolution with the Greens function. The metric $K e^{-s}$ on $V$ is flat, so for any $b$ there is a nonzero section $w$ of $V$ such that

$$
|w|_{K e^{-s}}=e^{-s / 2}|w|_{K} \leq|z|^{b} \text {. }
$$

In particular we may choose $b$ big enough so that

$$
|w|_{H} \leq e^{s / 2} \text {. }
$$

Aubin's estimate [2] is

$$
\int e^{s}<\infty
$$

since $\int|f|<2$. Thus $w$ is a section of $\bar{E} \cong \mathscr{O}_{X}^{r}$ which is holomorphic outside the origin, and is in $L^{2}$. This implies that it is holomorphic, so the subbundle $V$ has an extension over the puncture.

Corollary 10.7. In the situation of Theorem 3 , the $L_{1}^{2}$ subbundles of $E / X$ are exactly the restrictions of strict subsystems $V \subset a E$ on $X^{\mathrm{alg}}$.

Proof. By the lemma an $L_{1}^{2}$ subsystem comes from one over $X^{\text {alg }}$. On the other hand by the previous lemma, if $V \subset a E$ is a subsystem over $X^{\text {alg }}$ then the degree of $\left.V\right|_{X}$ is not $-\infty$. By the Chern-Weil formula this implies that $\left.V\right|_{X}$ is an $L_{1}^{2}$ subsystem.

Recall that a system $E$ with a metric $K$ is stable if

$$
\frac{\operatorname{deg}^{\mathrm{an}}(V)}{\mathrm{rk}(V)}<\frac{\operatorname{deg}^{\mathrm{an}}(E)}{\operatorname{rk}(E)}
$$

for every proper $L_{1}^{2}$ subsystem $V \subset E$. Analogously, say a regular system $E$ on $X^{\mathrm{alg}}$ is stable if

$$
\frac{\operatorname{deg}^{\mathrm{alg}}(V)}{\operatorname{rk}(V)}<\frac{\operatorname{deg}^{\mathrm{alg}}(E)}{\operatorname{rk}(E)}
$$

for every subsystem $V \subset E$.

Corollary 10.8. A system $E$ over $X$ with metric as in Theorem 3 is stable if and only if $a E$ is a stable system on $X^{\mathrm{alg}}$.

Proposition 3.3 implies that a system $a E$ which comes from a variation of Hodge structure is a direct sum of stable systems of degree zero. Corollary 10.8 gives information about what kinds of bundles can arise from variations of Hodge structure.

Lemma 10.9. Suppose $E^{\prime}$ and $E^{\prime \prime}$ are systems with metrics $K$ as in Theorem 3. Assume the metrics are flat. Then any bounded map $E^{\prime} \rightarrow E^{\prime \prime}$ is preserved by the connection.

Proof. This follows from Zucker's theorem [33], but we will give another proof. As in the proof of Theorem 3, it suffices to consider the case where the map is 
an isomorphism. Pull back the metric $K$ via the isomorphism, to get another metric $H=K h, h$ bounded. We have to show that $h$ is a flat section, or since it is selfadjoint, just that $D^{\prime \prime} h=0$. From the formula 3.1(c) we conclude that

$$
\Delta^{\prime} \operatorname{Tr}(h)=-\left|D^{\prime \prime}(h) h^{-1 / 2}\right|^{2} .
$$

The last part of Assumption 3 holds on $X$ so $\Delta^{\prime} \operatorname{Tr}(h)=0$ hence $D^{\prime \prime} h=0$.

Combining Proposition 2.4, Theorem 1, Proposition 3.3, Theorem 3, Corollaries 10.7, 10.8, and Lemma 10.9, we have proved

Theorem 4. Let $X$ be a quasiprojective curve. The construction ' $a$ ' of Theorem 3 gives an equivalence of categories from the category of complex variations of Hodge structure over $X$ to the category of regular systems of Hodge bundles on $X^{\mathrm{alg}}$ which are direct sums of stable systems of degree zero.

\section{REFERENCES}

1. L. V. Ahlfors, An extension of Schwarz's lemma, Trans. Amer. Math. Soc. 43 (1938), 359-364.

2. T. Aubin, Sur la fonction exponentielle, C. R. Acad. Sci. Paris 270A (1970), 1514-1516.

3. K. Corlette, Flat G-bundles with canonical metrics, J. Differential Geom. (to appear).

4. M. Cornalba and P. Griffiths, Analytic cycles and vector bundles on non-compact algebraic varieties, Invent. Math. 28 (1975), 1-106.

5. P. Deligne, Un théorème de finitude pour la monodromie, Discrete Groups in Geometry and Analysis, Birkhauser, Boston, 1987, pp. 1-19.

6. P. Deligne and J. Milne, Tannakian categories, Lecture Notes in Math., no. 900, Springer, New York, 1982, 101-228.

7. S. K. Donaldson, Anti self-dual Yang-Mills connections over complex algebraic surfaces and stable vector bundles, Proc. London Math. Soc. (3) 50 (1985), 1-26.

8. S. K. Donaldson, Infinite determinants, stable bundles, and curvature, Duke Math. J. 54 (1987), 231-247.

9. __ Twisted harmonic maps and the self-duality equations, Proc. London Math. Soc. (3) 55 (1987), 127-131.

10. P. Griffiths, Periods of integrals on algebraic manifolds I, II, Amer. J. Math. 90 (1968); III, Inst. Hautes Études Sci. Publ. Math. 38 (1970).

11. P. Griffiths et al., Topics in transcendental algebraic geometry, Princeton Univ. Press, Princeton, NJ, 1984.

12. P. Griffiths and W. Schmid, Locally homogeneous complex manifolds, Acta Math. 123 (1969), 253-302.

13. R. S. Hamilton, Harmonic maps of manifolds with boundary, Lecture Notes in Math., no. 471, Springer, New York, 1975.

14. N. J. Hitchin, The self-duality equations on a Riemann surface, Proc. London Math. Soc. (3) 55 (1987), 59-126.

15. M. Kashiwara, Vanishing cycles and holonomic systems of differential equations, Lecture Notes in Math., no. 1016, Springer, New York, 1983, pp. 134-142.

16. D. Kazhdan, On arithmetic varieties, Lie Groups and Their Representations, Halsted, New York, 1975.

17. R. Kobayashi, Einstein-Kähler metrics on open algebraic surfaces of general type, Tohoku Math. J. 37 (1985), 43-77. 
18. S. Kobayashi and T. Ochiai, Holomorphic structures modeled after hyperquadrics, Tohoku Math. J. 34 (1982), 587-629.

19. M. Lübke, Stability of Einstein-Hermitian vector bundles, Manuscripta Math. 42 (1983), 245257.

20. B. Malgrange, Polynôme de Bernstein-Sato et cohomologie évanescente, Astérisque 101-102 (1983), 243-267.

21. Y. Miyaoka, On the Chern numbers of surfaces of general type, Invent. Math. 42 (1977), 225237.

22. Y. Miyaoka, The maximal number of quotient singularities on surfaces with given numerical invariants, Math. Ann. 268 (1984), 159-171.

23. C. C. Moore, Compactifications of symmetric spaces II: the Cartan domains, Amer. J. Math. 86 (1964), 358-378.

24. D. Mumford and J. Fogarty, Geometric invariant theory, second ed., Springer-Verlag, New York, 1982.

25. M. S. Narasimhan and C. S. Seshadri, Stable and unitary bundles on a compact Riemann surface, Ann. of Math. 82 (1965), 540-564.

26. R. Palais, Foundations of global non-linear analysis, Benjamin, New York, 1968.

27. W. Schmid, Variation of Hodge structure: the singularities of the period mapping, Invent. Math. 22 (1973), 211-319.

28. B. Shiffman, Complete characterization of holomorphic chains of codimension one, Math. Ann. 274 (1986), 233-256.

29. C. Simpson, Yang-Mills theory and uniformization, Lett. Math. Phys. 14 (1987), 371-377.

30. K. K. Uhlenbeck and S. T. Yau, On the existence of Hermitian-Yang-Mills connections in stable vector bundles, Comm. Pure and Appl. Math. 39-S (1986), 257-293.

31. S. T. Yau, Calabi's conjecture and some new results in algebraic geometry, Proc. Nat. Acad. Sci. U.S.A. 74 (1977), no. 5, 1798-1799.

32. A. Weil, Introduction à l'étude des variétés kähleriennes, Hermann, Paris, 1952.

33. S. Zucker, Hodge theory with degenerating coefficients: $L^{2}$ cohomology in the Poincaré metric, Ann. of Math. 109 (1979), 415-476.

34. S. Kobayashi, Curvature and stability of vector bundles, Proc. Japan Acad. Ser. A 58 (1982), $158-162$.

35. M. Lübke, Chernklassen von Hermite-Einstein-Vektorbündeln, Math. Ann. 260 (1982), 133141.

36. V. B. Mehta and A. Ramanathan, Restriction of stable sheaves and representations of the fundamental group, Invent. Math. 77 (1984), 163-172.

37. F. Takemoto, Stable vector bundles on algebraic surfaces, Nagoya Math. J. 47 (1972), 29-48.

Department of Mathematics, Princeton University, Princeton, New Jersey 08544 